\title{
Recent Developments in Machine Learning for Energy Systems Reliability Management
}

\author{
Laurine Duchesne, Efthymios Karangelos, Member, IEEE, and Louis Wehenkel
}

\begin{abstract}
This paper reviews recent works applying machine learning techniques in the context of energy systems reliability assessment and control. We showcase both the progress achieved to date as well as the important future directions for further research, while providing an adequate background in the fields of reliability management and of machine learning. The objective is to foster the synergy between these two fields and speed up the practical adoption of machine learning techniques for energy systems reliability management. We focus on bulk electric power systems and use them as an example, but we argue that the methods, tools, etc. can be extended to other similar systems, such as distribution systems, micro-grids, and multi-energy systems.
\end{abstract}

Index Terms-Machine learning, reliability, electric power systems, security assessment, security control.

\section{INTRODUCTION}

A RTIFICIAL INTELLIGENCE (AI) emerged as a research subfield of computer science in the near aftermath of the second world-war, and started to expand towards software engineering in the 1970's. Recently, AI and more specifically Machine Learning (ML) has become a 'musthave' technology and a very active research field addressing complicated ethical and theoretical questions. This recent boom is facilitated by the continuous growth in the availability of computational power and advanced sensing and data communication infrastructures.

7 LECTRIC POWER SYSTEMS (EPS) emerged during

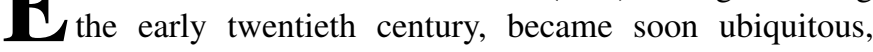
and progressively more and more computerised since the 1970's. Recently, EPS started to undergo a revolution, in order to respond to societal and environmental challenges; renewable energy sources, micro-grids, power electronics, and globalisation are indeed changing their game. The changes characterising such revolution are pushing the existing analytical methods for power system reliability assessment and control to their limits.

The first proposals for applying ML to EPS dynamic security assessment and control (a part of EPS reliability management) were already published during the 1970's and 1980's [1]. In spite of a significant number of academic publications since then, only a few real-world applications have been reported. This should be contrasted by the very significant practical impact of control, simulation, optimisation, and estimation theories on EPS engineering, and in particular on their reliability management. Recently, research on the application of ML to EPS reliability management has

LD, EK and LW are with the Montefiore Institute of Research in Electrical Engineering and Computer Science, University of Liège, 4000 Liège, Belgium. E-mails: \{L.Duchesne, E.Karangelos, L.Wehenkel\}@uliege.be. experienced a very vivid revival. This is hopefully not only explained by the trendy behaviour of the research community and funding agencies, but rather by the fact that new ideas and techniques are available and liable to increase the potential for real-world impact.

This paper seeks to foster the synergy between the electric power and energy systems and machine learning communities and enable further work both by industry and academia, in order to speed up the practical adoption of machine learning techniques for energy systems reliability management. To do so, we analyse the recent machine learning applications for electric power system reliability management over the past 5 years. We focus on showcasing both the progress achieved to date as well as the important future directions for further research. In order to address audiences from both communities, we briefly provide an adequate background in the fields of reliability management and of machine learning. Finally, while we focus here on the electrical power systems, we also discuss how the progress with the use of machine learning applications in this field can be the blueprint for applying machine learning in the broader context of other energy systems, such as distribution systems, micro-grids, and multi-energy systems.

The rest of this paper is organised as follows: sections II and III synthetically present the required background in reliability management and in machine learning; section IV provides statistics about the publications of ML applied to EPS reliability management since the year 2000, while sections V, VI and VII review published works over the last 5 years. Section VIII discusses open problems and directions for future work in the context of distribution systems, micro-grids and multi-energy systems reliability management.

\section{RELIABILITY MANAGEMENT BASICS}

In this section we introduce electric power systems reliability management, to set the background for sections IV-VI, in the interest of readers outside the electric power systems community. In particular, we introduce the decisions, time horizons and uncertainties related to reliability management, the differences between security and adequacy, as well as between static and dynamic security, and finally the functions of reliability assessment and reliability control and the current challenges to tackle these tasks.

\section{A. Types of decisions, time horizons \& uncertainties}

In general terms, (electric power) system reliability expresses a level of confidence in providing a continuous supply 


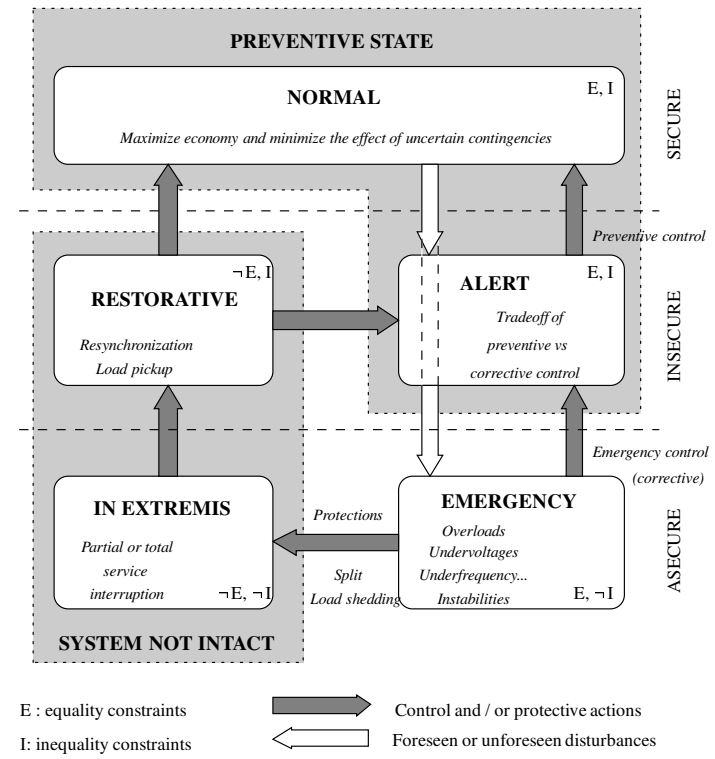

Fig. 1. State transition diagram for security control (Adapted from [2])

(of electricity) to the system end-users. Reliability management concerns taking decisions to ensure that, in spite of uncertainties, the system reliability shall be suitable over some specified future time horizon.

With a long-term perspective (indicatively, 10-30 years in advance), the main decision is to define the additional infrastructure investments needed to keep the future system reliable enough. Next, in a mid-term context (typically 15 years ahead in time), the prevailing question is how to maintain the functionality of the existing system through repairs and/or replacements of its individual components. Last, but certainly not the least, short-term operation planning (a few months to a few hours ahead) and real-time operation jointly aim at deciding how to optimally deliver electricity from the producers to the end-users while enabling equipment maintenance and infrastructure building activities.

Various types of uncertainties and various spaces of candidate decisions challenge all these complex and large-scale decision making problems. Better uncertainty modelling, enhanced probabilistic and/or robust decision making frameworks, and more effective algorithms for optimal decision making under uncertainties are therefore main directions of R\&D in electric power systems reliability management.

\section{B. Adequacy \& security}

From a functional standpoint, power system reliability can be sub-divided into adequacy and security [3], respectively defined as,

Adequacy - the ability to supply with high enough probability the end-users at all times, taking into account outages of system components;

Security - the ability to withstand sudden disturbances such as electric short circuits or nonanticipated loss of system components without major service interruptions.

A reliable power system thus exhibits both (i) redundancy to adequately supply the load demand even when some of its components remain unavailable, and (ii) plasticity to securely ride-through sudden, unanticipated disturbances and/or disconnections of some of its components.

Adequacy emphasises on the system dimensioning to accommodate the variability and stochasticity of the end-user demand, while also taking into account the (random) unavailabilities of system components. Typically, (in)adequacy is evaluated over a period of time, ranging from a few months to many years, and expressed by indicators such as loss of load probability or expected energy not supplied [3]. It may also be quantified in terms of the socio-economic impact of service interruptions to the system end-users, through indicators such as the expected cost of energy not supplied. Explicit adequacy criteria are commonly used in long-term planning applications adopted by many system operators [4].

Security complements adequacy by focusing on the operation of the system while it undergoes state transitions initiated by unexpected exogenous disturbances and is canalised by various preventive or corrective control actions ( $c f$. "The adaptive reliability control system" of T. Dy Liacco [5]). The diagram shown in Fig. 1, originally introduced in [2] based on a simpler version already given in [5], depicts the transitions among power system states from the security perspective. Security assessment aims at determining at which security level the system is currently residing, whereas security control aims at deciding control actions to move towards a more secure state. The vast domain of security (assessment and/or control) is further decomposed into dynamic and static security (assessment and/or control).

Dynamic security characterises the ability of the system to complete the transition from the pre-disturbance operating state to a post-disturbance stable equilibrium state. Here, three main physical phenomena are at (inter)play, giving rise to respective classes of (in)stability, namely rotor-angle, voltage and frequency (in)stabilities [6]. We refer the reader to the textbook of Kundur [7] for an explanation of the physical and mathematical modeling properties of these phenomena.

Rotor-angle stability concerns the equilibrium between the mechanical (input) and electromagnetic (output) torque of synchronous generators, keeping all machines of an interconnected power system rotating at a common angular speed. It is further classified into small-signal and transient rotor-angle stability, according to the magnitude of the studied disturbances. Transient rotor-angle stability concerns the ability to sustain large disturbances such as short-circuits followed by the disconnection of one or more transmission lines, whereas small-signal stability concerns the ability to absorb stochastic variations of demand and generation. The physics of both concern relatively fast dynamics ranging over a few seconds following a disturbance.

Voltage stability refers to the ability of the power system to avoid an uncontrollable deterioration of the voltage level at its buses. At the extreme, voltage collapse is the situation wherein the system bus voltages reduce to unacceptably low levels. The behaviour of electrical loads and tap-changing voltage transformers restoring the consumed power after a disturbance is the main phenomenon potentially driving the system to voltage collapse. The dynamics of these phenomena 
are typically slower than those concerning rotor-angle stability, and they may range over several minutes.

Frequency stability concerns the ability of the system to contain the frequency deviations caused by large mismatches between generation and demand resulting for example from the loss of large generators or fast variations of the load. Frequency stability is an issue of major concern under islanded operation, following some event that results in splitting the interconnected power system into disconnected under-/overgenerated sub-parts. In systems with low electromechanical inertia, such as systems with predominantly photovoltaic and inverter-connected wind-power generation systems, frequency (in)stability is likely to become a major problem. While loss of synchronism typically takes at most a few seconds, frequency instability may take up to a few minutes to develop, and its study thus requires the modeling of slower processes such as boiler dynamics and load recovery mechanisms.

Finally, static security characterises the viability (typically over a period of $5-60$ minutes) of the steady-state reached by the system following a contingency (i.e. a sudden line, transformer or generator outage). The main physical aspect of interest is compliance with the permanent capabilities of the system components (e.g. current carrying or electric isolation capabilities). Static Security Assessment (SSA) can be carried out by using a Power Flow (PF) solver to calculate a postcontingency state for each element of a set of contingencies. In current practice the so-called "N-1" contingency set is most often used: it is the set of disturbances corresponding to singlecomponent trippings ("N" denotes the total number of such components). On the other hand, the Optimal Power Flow (OPF) problem may be solved in order to determine least cost decisions making a steady state viable.

\section{Reliability assessment vs reliability control}

Managing the aforementioned aspects to ensure the reliable supply of electricity is in practice achieved through the functions of reliability assessment and reliability control. Reliability assessment concerns evaluating the security and adequacy metrics necessary to assess whether the system reliability level is acceptable with respect to a certain reliability criterion. It can be performed ex-ante to determine whether taking a certain candidate decision suffices to achieve the reliable operation of the system over a future horizon, or $e x$ post to evaluate the effect of already taken decisions over some past operational period. Reliability control concerns finding the decisions so as to ensure that the system will comply with a certain reliability criterion, and while optimising a socio-economic objective [8]. The formalisation of reliability assessment and control problems, as well as the challenges for tackling these, depend on the precise reliability management context of interest.

Starting from the shortest horizon, in the context of real-time operation, the salient feature is the lack of computational time to simulate the behaviour of the system in the time-domain, re-evaluate the static operability of the system vs credible contingencies and search for appropriate remedial actions. It is thus necessary to rely on security rules prepared off-line to classify the system dynamic security, while monitoring its operation and its compliance with static security limits. Similarly, emergency controls need to be implemented as soon as possible to contain the unwanted deterioration of the system state before it escalates, and thus can only be triggered on the basis of pre-defined strategies. The challenge is therefore to design simple yet robust assessment rules and closed-loop control strategies, while also taking into account the reliability of protection, control and communications infrastructures.

In the short-term operation planning context, reliability management is further complicated by the need to take into account (i) the uncertainty on the potential pre-disturbance operating state of the system and the temporal evolution thereof, and, (ii) the future remedial actions to be implemented as per the respective real-time operation strategies. Analytical methods, such as time-domain simulations for dynamic security, power flow and optimal power flow for static security and adequacy are the primary tools for reliability assessment. The approach boils down to using such tools over a representative sample of potential operating conditions, to compute estimates of the respective metrics. The challenge is of course related to the size of the sample needed to reach acceptable accuracy. The Security Constrained Optimal Power Flow (SCOPF) is the fundamental statement expressing the operation planning reliability control problem, focusing mostly on static security [9][11]. Different variants of this problem are cast under different assumptions to fit specific operation planning questions (e.g. from the linear, so-called Direct Current or DC approximations employed in market-based generation dispatching to the optimisation of preventive/corrective actions under the nonlinear Alternating Current or AC power flow model). Further from the dimensionality issues associated with injection (i.e. load and generation) uncertainties, a key issue here is how to effectively integrate dynamic security limitations in the framework of such problem.

Taking the mid-term asset management perspective, the central question for reliability assessment concerns the criticality of a certain asset for the power system functionality, with emphasis on the adequacy and static security aspects. Answering this question entails essentially simulating the operation of the system with and without the asset in question, using again power flow and optimal power flow methods in a Monte Carlo style approach. Reliability control seeks to identify which assets to maintain and when to do so. Componentbased reliability rules, triggering maintenance activities by age, condition, maintenance frequency etc. are useful in practice to answer the former question, taking into account the large number of system components. The problem of maintenance scheduling includes logistical considerations on top of the criticality of assets for the network functionality. Such logistical considerations reduce the (theoretically large) set of potential maintenance schedules in a calendar year, to a smaller subset of alternative moments per component in question. Still, the scheduling question implies a large-scale stochastic mixedinteger programming problem and the typical approach is to use heuristics for finding a suitable moment for each prioritised maintenance activity, while minimising the impact on reliable operation. 
Finally, given the vast uncertainties in a long-term horizon, reliability management can only be achieved by a recursive approach integrating assessment and control. The first step entails identifying the needs of the future power system, employing both adequacy/static security tools in Monte Carlo simulations as well as dynamic security consideration to frame potential future reliability problems. Exhaustive search is far from being an option here and the challenge is to combine microand macro- assumptions to generate manageable subsets of future operational conditions. Based on identified problems, expert knowledge as well as considerations on project timeline feasibility, public acceptability, etc. are employed to arrive at a small, manageable subset of potential solutions to the identified needs. These solutions then need to be re-assessed over a new set of realisations from the uncertainty models while the final choice requires detailed study of both the static and the dynamic behaviour of the resulting grid, as well as socio-economic analysis with a view on electricity markets and on the impact on the natural environment.

\section{MACHINE LEARNING CONCEPTS}

Machine learning exploits data gathered from observations or experiments on a system to automatically build models predicting or explaining the behaviour of the system, or decision rules to interact in an appropriate way with it. In this section we introduce the basic concepts of this field, to serve as the background for sections IV-VI, in the interest of readers outside the machine learning community. In particular, we introduce the different types of machine learning problems with a focus on supervised learning, feature selection and engineering, how to choose a method, and how to assess the accuracy of a model.

\section{A. Different types of machine learning problems}

To introduce the main types of machine learning problems, we will use the probabilistic/statistical formalisation and terminology and restrict to the so-called batch-mode setting. We refer the interested reader to more general textbooks for further information [12]-[14].

1) Supervised learning (SL): Given a sample $\left\{\left(x^{i}, y^{i}\right)\right\}_{i=1}^{n}$ of input-output pairs, a (batch-mode) supervised learning algorithm aims at automatically building a model $\hat{y}(x)$ to compute approximations of outputs as a function of inputs.

The standard probabilistic formalisation of supervised learning considers $x \in X$ and $y \in Y$ as two (vectors of) random variables drawn from some (joint) probability distribution $P_{x, y}$ over $X \times Y$, a real-valued loss function $\ell$ defined over $Y \times Y$, and a hypothesis space $\mathcal{H}$ of "predictors" (i.e. functions from $X$ to $Y$ ), and measures the inaccuracy (named the expected loss, or average loss, or risk) of a predictor $h \in \mathcal{H}$ by

$$
L(h)=E\{\ell(y, h(x))\}=\int_{X \times Y} \ell(y, h(x)) d P_{x, y} .
$$

Denoting by $(X \times Y)^{*}$ the set $\bigcup_{n=1}^{\infty}(X \times Y)^{n}$ of all finite size samples, a (deterministic) supervised learning algorithm $A$ can thus formally be stated as a mapping

$$
A:(X \times Y)^{*} \rightarrow \mathcal{H}
$$

from $(X \times Y)^{*}$ into a hypothesis space $\mathcal{H}$. Statistical learning theory studies the properties of such algorithms, in particular how well they behave in terms of loss $L$ when the sample size $n$ increases [12].

Since SL is the most common type of machine learning problem treated in reliability management, we describe in more details the characteristics of SL in section III-B.

Methods: Methods like decision trees, neural networks, linear regression, nearest neighbor, support vector machines etc. belong to this category.

Power system example: A first example application is the assessment of system stability after the occurence of a disturbance [15]. In that case, the target output $y$ typically can take two values, stable or unstable, and the inputs $x$ could be real-time measurements of voltage at each bus and power flow in each branch of the system. The problem then amounts to building a model $\hat{y}(x)$ that predicts, based on the measurements, if the system is stable or unstable.

2) Reinforcement learning (RL): Given a sample of $n$ trajectories of a system

$$
\left\{\left(x_{\tau_{i}}^{i}, d_{\tau_{i}}^{i}, r_{\tau_{i}}^{i}, x_{\tau_{i}+1}^{i}, \ldots, d_{\tau_{i}+h_{i}-1}^{i}, r_{\tau_{i}+h_{i}-1}^{i}, x_{\tau_{i}+h_{i}}^{i}\right)\right\}_{i=1}^{n},
$$

batch-mode reinforcement learning aims at deriving an approximation of an optimal decision strategy $\hat{d}^{*}(x, t)$ maximising system performance in terms of a cumulated index (named reward) over a certain horizon $T$, defined by

$$
R=\sum_{t=0}^{T-1} \gamma^{t} r_{t}
$$

where $\gamma \in(0,1]$ is a discount factor. In this framework, $x_{t}$ denotes the state of a dynamic system at time $t, d_{t}$ is the control decision applied at time $t$, and $r_{t}$ is an instantaneous reward signal [14].

From a theoretical point of view, reinforcement learning can be formalised within the stochastic dynamic programming framework. In particular, supposing that the system obeys to time invariant dynamics

$$
x_{t+1}=f\left(x_{t}, d_{t}, w_{t}\right),
$$

where $w_{t}$ is a memoryless and time invariant random process and obtains a bounded time invariant reward signal

$$
r_{t}=r\left(x_{t}, d_{t}, w_{t}\right),
$$

over an infinite horizon $(T \rightarrow \infty)$, one can show that the two following (Bellman) equations define an optimal decision strategy

$$
\begin{gathered}
Q(x, d)=E\left\{r(x, d, w)+\gamma \max _{d^{\prime}} Q\left(f(x, d, w), d^{\prime}\right)\right\}, \\
d^{*}(x)=\arg \max _{d} Q(x, d) .
\end{gathered}
$$

Reinforcement learning can thus be tackled by developing algorithms to solve these equations approximately when the sole information available is provided by a sample of system trajectories. The theoretical questions that have been studied in this context concern the statement of conditions on the sampling process and on the learning algorithm ensuring convergence to an optimal policy in asymptotic conditions (i.e. when $n \rightarrow \infty)$. 
Methods: Methods such as Q-learning, State-ActionReward-State-Action (SARSA), and more recently Deep Q Network (DQN) belong to this category.

Power system example: A possible application of reinforcement learning in power systems is emergency control, where an RL algorithm could take a sequence of actions $d_{t}$ when the system is in an emergency state in order to come back to a normal state, as in [16]. Other examples can be found in [17].

3) Unsupervised learning (UL): Given a sample of observations $\left\{z^{i}\right\}_{i=1}^{n}$ obtained from a certain sampling distribution $P_{z}$ over a space $Z$, the objective of unsupervised learning is essentially to determine an approximation of the sampling distribution. In the most interesting case, $Z$ is a product space $Z_{1} \times \cdots \times Z_{p}$ defined by $p$ discrete or continuous random variables, and the main objective of unsupervised learning is to identify the relations among these latter, such as (conditional) independence relations or colinearity relations, as well as the parameters of their distributions.

Methods: Earlier work in this field concerned clustering (for instance with the $k$-means algorithm), Principal Component Analysis (PCA) and hidden Markov models. More recent research topics, still very active today, concern independent component analysis as well as the very rich field of graphical probabilistic models, such as Bayesian belief networks [13].

Power system example: In the context of powers systems reliability, unsupervised learning can be used for segmenting automatically large scale power grids into coherent zones to ease the management of the grid for control room operators [18].

4) Semi-supervised learning: Semi-supervised learning concerns a situation where the dataset is composed of a labelled sample $\left\{\left(x^{i}, y^{i}\right)\right\}_{i=1}^{n}$ drawn from a joint distribution $P_{x, y}$ and a second (unlabelled) sample $\left\{\left(x^{j}\right)\right\}_{j=1}^{n^{\prime}}$ drawn from the corresponding marginal distribution $P_{x}$. Semi-supervised learning algorithms aim at exploiting both samples together to find a predictor $h$ that is hopefully more accurate than what could be produced by a supervised learning algorithm using only the labelled sample.

These types of methods are useful when it is relatively 'easy' to collect unlabelled data but relatively 'difficult' to obtain labelled data.

Methods: Methods based on self-training, tri-training and other algorithms such as semi-supervised support vector machines belong to this category.

Power system example: In the context of dynamic security assessment, one could use time-consuming simulations to label as stable or unstable only a part of a database, and then use semi-supervised learning to exploit the whole database in order to build a classifier, as in [19].

\section{B. Characteristics of supervised learning algorithms}

In supervised learning a first main distinction concerns the nature of the output space. When $Y$ is a finite set of 'class labels' one talks about classification problems (e.g. stable vs unstable), while when $Y$ is embedded in the set of real numbers (respectively in a finite dimensional euclidean space) one talks about regression (respectively multiple-regression) problems (e.g. stability margin). But, beyond these two standard types of supervised learning problems, there are many other ones, as many as one can imagine output spaces structured in some meaningful way.

Once an output space $Y$ is defined, one can further refine the nature of the problem by defining a particular loss function $\ell\left(y, y^{\prime}\right)$ over $Y \times Y$. For example, for classification problems one often uses the so-called 0/1-loss, which is defined by $\ell\left(y, y^{\prime}\right)=1\left(y \neq y^{\prime}\right)$ and counts the number of misclassifications, whereas for regression problems one often uses the square-loss $\left(y-y^{\prime}\right)^{2}$. Once the loss function $\ell$ is defined, it means that one targets the so-called "Bayes" model, which is defined in a point-wise way by

$$
h_{B}(x)=\arg \min _{y^{\prime} \in Y} \int_{Y} \ell\left(y, y^{\prime}\right) d P_{y \mid x},
$$

and is among all functions $h$ of $x$ the one minimising the average loss $L(h)$, defined as in (1). For example, in the case of regression problems and with the square-loss, $h_{B}(x)$ is identical to the conditional expectation of $y$ given $x$, whereas using the absolute loss $\left|y-y^{\prime}\right|$ would instead yield the conditional median.

The next step in defining a supervised learning algorithm consists of choosing a hypothesis space of functions $\mathcal{H}$. As far as loss minimisation is concerned, this space should ideally contain the (problem-specific) Bayes model or at least models sufficiently close to it in terms of the chosen loss function.

Given a choice of $\mathcal{H}$, the empirical risk minimisation principle then reduces supervised learning to solving the following minimisation problem:

$$
A\left(\left\{\left(x^{i}, y^{i}\right)\right\}_{i=1}^{n}\right)=\arg \min _{h^{\prime} \in \mathcal{H}} \sum_{i=1}^{n} \ell\left(h^{\prime}\left(x^{i}\right), y^{i}\right) .
$$

One can show that if $\mathcal{H}$ is 'sufficiently' small, it produces models whose loss $L$ converges towards $\min _{h \in \mathcal{H}} L(h)$, when the sample size $n$ increases, and that it converges faster if $\mathcal{H}$ is of smaller 'size'. As far as accuracy is concerned, the SL algorithm should thus use an as small as possible hypothesis space containing good enough approximations of $h_{B}$.

Nevertheless, in addition to accuracy, computational complexity of learning algorithms is often a concern. Indeed, solving an empirical loss minimisation problem may require huge computational resources if the hypothesis space is very complex and the sample size $n$ is very large.

Finally, beyond making 'low loss' predictions, the goal of a machine learning application is often to help human experts to understand the main features of the problem at hand. Therefore, interpretability of the output of the machine learning algorithm is another often highly desired feature.

\section{Feature selection and feature engineering}

When considering a particular application of machine learning, the raw datasets that are available are typically recorded values of a large number of low-level individual variables $z_{j}$, some of which could be either inputs $x_{j}$, outputs $y_{k}$, or decisions $d_{l}$ of some supervised and/or reinforcement learning problem of interest. 
Often, it is suspected that some (or even a majority) of these variables are actually irrelevant for the resolution of some particular prediction or optimal control task. In other applications, one would like to find a minimal subset of input variables to be used by a supervised or reinforcement learning method, so as to facilitate the practical application of the resulting predictor or control policy without sacrificing too much in terms of accuracy.

Thus, the machine learning field has developed various methods for selecting "optimal" subsets of input variables (feature selection) and for computing useful functions of the original variables (feature extraction), based on the information provided in a dataset [20].

\section{Practical choice of a supervised learning method}

The different supervised learning algorithms available today (see [12], [13]), yield different compromizes between interpretability, computational efficiency, and accuracy.

The choice of the most suitable algorithm is thus highly application dependent, for several reasons: (i) the application determines which one of these three criteria is the most important one in practice; (ii) the application determines the data-generating mechanism and loss function, hence the Bayes model $h_{B}$ and thus how well different hypothesis spaces allow to approximate this ideal target predictor; (iii) the application domain conditions the size of the possibly available datasets, impacting both accuracy and computational efficiency of most algorithms, but in different ways.

This situation means that, typically, the one who is faced with a particular application will try out a (more or less large) subset of machine learning algorithms, analyse their behaviour and results, and select the one which seems to fit in the best way the need of the considered application. This "trial and error" type of approach is the price to pay for the very broad scope of machine learning applications.

\section{E. Overfitting and honest model assessment}

Because the empirical risk minimisation chooses models to minimise the average loss over the learning sample, this empirical risk is in practice strongly biased in an optimistic way. It means that even if the selected predictor works very well on the learning sample, there is no guarantee that it will work well also on an independent test sample drawn from the same distribution.

In order to assess the accuracy in an honest way, various approaches have been developed and studied, such as the "hold-out" method which keeps part of the data as a test sample and uses only the rest of them to apply the learning algorithm, or the $k$-fold cross-validation approach, which uses the data in a more effective way at the price of higher computational requirements [12].

Often, the cross-validation approach is also used in order to select among several algorithms the one more suited to a particular dataset, or to adapt some algorithm's "meta-parameters" (e.g. number of hidden neurons, training epochs, strength of weight decay penalisation), or even to help identifying a subset of relevant input features. If this is the case, then a nested model assessment approach is needed in order to honestly assess the accuracy of the finally produced predictor [12].

\section{Publication statistics Since 2000}

In this section, we survey recent contributions to the field of machine learning for electric power systems reliability. We found (via Google Scholar and Scopus) ${ }^{1} 366$ papers in this subject field that were published between January 2000 and October 2019; their yearly counts are shown in Figure 2, which clearly shows a strong growth over the last 5 years.

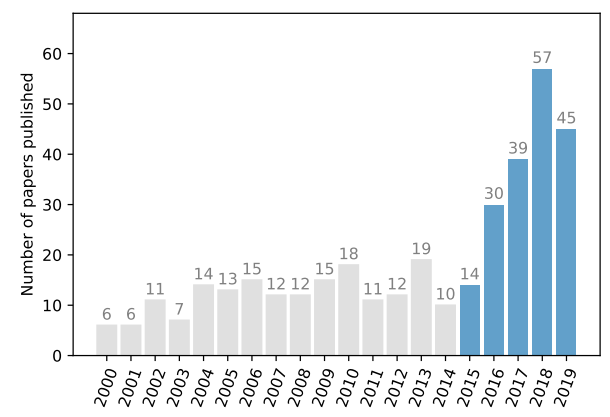

Fig. 2. Yearly number of published papers we found on the topic of machine learning application to electric power systems reliability

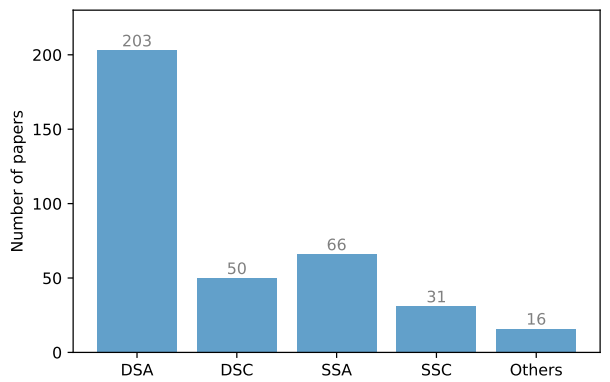

Fig. 3. Number of ML papers (01/2000 - 10/2019) per reliability management problem $(\mathrm{DSA}=$ dynamic security assessment, $\mathrm{DSC}=$ dynamic security control, $\mathrm{SSA}=$ static security assessment, $\mathrm{SSC}=$ static security control)

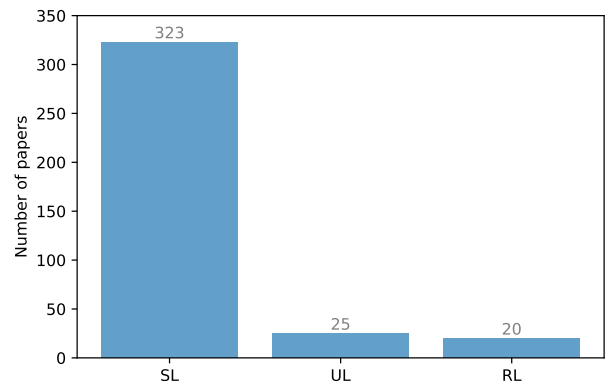

Fig. 4. Number of papers per ML protocol (01/2000 - 10/2019)

Figure 3 shows the statistics of these papers in terms of the different types of reliability management problems they address. We observe that more than $50 \%$ of them address the problem of dynamic security assessment. On the other hand,

\footnotetext{
${ }^{1}$ We used the following keywords to gather these papers: i. power system, reliability, security, stability, learning; ii. power system, reliability, security, stability, assessment, control, learning; iii. power system, reliability, security, stability, assessment, control, learning, neural network, ANN, support vector, SVM, nearest neighbours, decision tree, random forest. The retrieved papers were analysed one by one to eliminate irrelevant ones from the statistics.
} 
we have observed a recent growth of the number of papers applying machine learning to power flow and optimal power flow computations (which are classified into the SSA and SSC categories respectively).

Another interesting analysis is the type of machine learning protocol exploited in our set of papers. Figure 4 shows the number of papers using supervised learning, unsupervised learning and reinforcement learning. It is clear that supervised learning is by far the most popular type of machine learning protocol used in these reliability management applications.

In the following two sections, we present in more details how the different reliability management problems are addressed in the literature with a machine learning approach. We first present papers exploiting machine learning for dynamic security assessment, as well as (dynamic) emergency and preventive control, since they constitute the largest part of our survey. Then we present how machine learning was applied for static security assessment and control, and to speed up optimal power flow and power flow computations. We focus this literature survey on the last 5 years. Table I presents an overview of the works discussed in the next two sections, classified in terms of the respective power system reliability management application (i.e. transient, voltage, frequency or small-signal stability, dynamic and static security).

\section{WORKS ON ML FOR DSA \& DSC SINCE 2015}

Dynamic security is a particularly suitable application for machine learning, given the need for fast assessment and control and the computational burden inherent to classical approaches such as time-domain simulations. Figure 5 shows the four steps generally followed to exploit machine learning in this context. We discuss them one-by-one hereafter.

\section{A. Database building}

The first step to apply machine learning for security assessment and control is the database building. In most papers, due to lack of historical data availability, simulations are used to generate a security database. Another advantage of simulation is that it allows to sample operating points defining well the security boundary, which is typically not the case with historical data, for which most operating points are stable [21]. The database generation is then usually done offline, given the extensive simulation cost to build it, while the application of the resulting model trained on the dataset can be done offline or online, depending on the application and the context.
TABLE I

MAIN SECURITY PROBLEMS ADDRESSED WITH A MACHINE LEARNING APPROACH AND CORRESPONDING REFERENCES

\begin{tabular}{|c|c|}
\hline Security problems addressed & References \\
\hline & {$[15],[16],[19],[22],[27]-[29]$,} \\
& {$[32],[34],[35],[37],[38],[41]$,} \\
{$[43],[45],[47],[49]-[52]$,} \\
Transient stability & {$[54]-[56],[63],[64],[67]-[73]$,} \\
& {$[75],[76],[81]-[83],[85]-[88]$,} \\
& {$[90],[92],[93],[98],[102],[105]$,} \\
{$[107],[113],[115]-[117]$} \\
\hline Voltage stability & {$[26],[30],[39],[40],[42],[44]$,} \\
& {$[46],[48],[53],[60],[65],[66]$,} \\
& {$[78],[79],[89],[91],[97],[100]$,} \\
& {$[103],[108]-[110],[114],[123]$} \\
\hline Small-signal stability & {$[21],[22],[61],[62],[74],[120]$,} \\
& {$[121]$} \\
\hline Frequency stability & {$[80],[84],[104],[106],[112]$} \\
\hline & {$[25],[31],[33],[36],[57]-[59]$,} \\
\hline & {$[77],[94],[95],[99],[101],[118]$,} \\
& {$[119],[122]$} \\
\hline Dynamic security & {$[18],[23],[24],[74],[96],[111]$,} \\
& {$[120],[121],[124]-[175]$} \\
\hline \multirow{2}{*}{ Static security } &
\end{tabular}

In order to generate a database based on simulations, the first step is to generate representative operating states of the system. The main uncertainties in power systems relate to load patterns, topology configuration and generation. It is impossible to sample all operating conditions and therefore Monte-Carlo simulations are used in most papers, but other techniques to sample from a multi-dimensional distribution are possible, such as the latin hypercube sampling [22].

In DSA and DSC applications, the input-features of the database often come from Phasor Measurement Units (PMU), which are devices allowing to monitor the power system state in real-time. They measure synchronously voltage phasors at buses where they are located and current phasors in the branches connected to these buses.

The target output of the database depends on the task. Most studies build classifiers to predict the stability status of the system, considering either transient, small-signal, voltage or frequency stability. Others are more interested in quantifying the distance to instability, by exploiting regressors to predict for instance the security margin, the voltage stability index, the so-called Critical Clearing Time (CCT), i.e. the maximum time available to clear a disturbance before the system becomes unstable, etc. Finally, for control purposes, some predict directly which decisions, such as generation re-dispatch, to

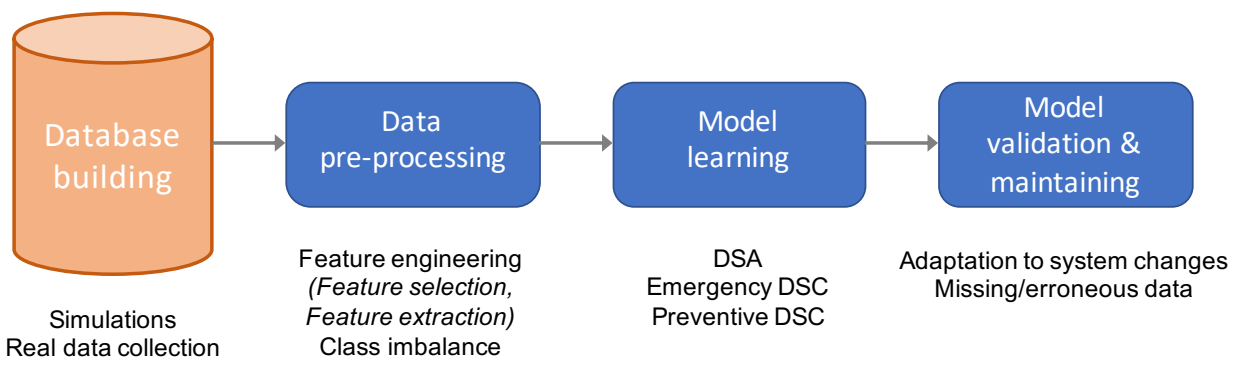

Fig. 5. Usual steps followed to exploit machine learning in DSA \& DSC 
apply. These outputs are usually obtained with time-domain simulations of the power system.

The quality and representativity of the database has a major impact on the effectiveness of the ML approach. For instance, for a database based on simulation, if the representation of the problem is too simple, this could lead to a learnt model with very good performance on data generated with the same distribution as the database but bad performance when it is used in practice, in a real situation. Furthermore, the input variables must allow one to discriminate well the target output(s). If it is not the case, the problem may be hard to learn and one may overfit noise, which could lead to difficulties to obtain an efficient model. The database must also be large enough for the model to be able to capture all the subtleties of the studied problem, in order to obtain high generalisation performances. Another important aspect for the person exploiting the database is to know the hypotheses used to generate it, if this is a database based on simulations, and to know the data collection process, if if it is based on real-life data. In general, when the quality and/or representativity of the database is insufficient, the learnt models cannot be used to perform security assessment and/or control in practice.

Recently, given the importance of the database generation step, papers focusing mainly on building more effective databases for machine learning-based security assessment and control were published [21], [23], [24]. In [23], the authors propose an approach using Vine-Copulas to generate more representative states of power systems. They show on a testcase that the security classifier built with this approach is superior to the one build with data obtained from a classical approach. Thams et al. exploit in [21] convex relaxation techniques and complex network theory to discard large parts of the input space and thus focus on the regions closer to the security boundary. In order to build a database representing well the security boundary, they use a 'Directed Walks' algorithm to identify the security boundary. Finally, [24] proposes a method to generate datasets to characterise the security boundary which cover equally the secure and insecure regions. In particular, they introduce infeasibility certificates based on separating hyperplanes to identify, for large portions of the input space, the infeasible region.

Furthermore, to help dealing with a massive amount of data, in [25], the authors propose a distributed computing platform for data sampling, feature selection, knowledge discovery and online security analysis.

\section{B. Data pre-processing}

Once a dataset is built, the data can be processed before being fed to a learning algorithm. This step may in some cases be mandatory. Processing the data can improve the quality of the predictions of learning algorithms, increase training speed and transform data in more meaningful representation to facilitate model training. In this section, we distinguish feature engineering and class imbalance management. Table II provides an overview of the main data pre-processing methods used for DSA and DSC discussed below.
TABLE II

MAIN DATA PRE-PROCESSING METHODS USED FOR DSA \& DSC OVER THE LAST FIVE YEARS AND CORRESPONDING REFERENCES

\begin{tabular}{|c|c|c|c|}
\hline \multicolumn{3}{|c|}{ Methods } & References \\
\hline \multirow{8}{*}{$\begin{array}{c}\text { Feature } \\
\text { engineering }\end{array}$} & \multirow{4}{*}{$\begin{array}{c}\text { Feature } \\
\text { selection }\end{array}$} & Filter methods & {$[22],[26]-[36]$} \\
\hline & & Wrapper methods & [34], [35], [37] \\
\hline & & Genetic algorithms & [38], [39] \\
\hline & & $\begin{array}{l}\text { Tree-based } \\
\text { algorithms }\end{array}$ & {$[40]-[43]$} \\
\hline & \multirow{4}{*}{$\begin{array}{l}\text { Feature } \\
\text { extraction }\end{array}$} & PCA and variants & [44]-[46] \\
\hline & & $\begin{array}{c}\text { Fisher's linear } \\
\text { discriminant }\end{array}$ & [47] \\
\hline & & $\begin{array}{l}\text { Shapelets for time } \\
\text { series }\end{array}$ & [48] \\
\hline & & $\begin{array}{l}\text { Deep learning } \\
\text { auto-encoders }\end{array}$ & {$[49]-[52]$} \\
\hline \multirow{3}{*}{\multicolumn{2}{|c|}{$\begin{array}{c}\text { Class } \\
\text { imbalance }\end{array}$}} & Oversampling & {$[24],[53],[54]$} \\
\hline & & $\begin{array}{l}\text { Cost-sensitive } \\
\text { learning }\end{array}$ & {$[53],[55]$} \\
\hline & & Ensemble methods & $\begin{array}{c}{[41],[45],[56],} \\
{[57]}\end{array}$ \\
\hline
\end{tabular}

1) Feature engineering: Given the large number of features necessary to fully describe the state of a power system and the need for fast algorithms, feature selection techniques are proposed in many papers. Too many features can lead to excessive training time and, if many features are not relevant, could decrease the performance of the learnt model. In the machine learning literature, several techniques have been proposed to reduce the number of features. In power systems applications, the 'Relief' method, which is is a filter-based method, has been used alone [22], [26], or combined with a PCA to reduce even more the number of features [27], [28]. Variants of this method such as 'Relief-F' have also been used [29]-[33], mostly to improve the predictions of randomised learning algorithms such as extreme learning machines.

Combining both filter and wrapper methods for feature selection, Zhou et al. [34] use the improved 'Relief-Wrapper' algorithm to select a subset of features and in [35], the authors propose a hybrid filter-wrapper feature selection algorithm using the 'Relief-F' method to find top weighted features and then the Sequential Floating Forward Selection method to select the most relevant set. In [37] a backward feature selection approach is used, on the other hand.

Genetic Algorithms (GAs) can also be used to select features. In [38], the authors use Particle Swarm Optimization (PSO) based feature selection. Packets of features are drawn randomly with PSO and the selected packet is the one maximising the mean of the standard deviations of the packet. In [39], the authors first apply a mutual information criterion to remove less significant features from the dataset and then use a multi-objective biogeography-based optimisation program to keep the most relevant ones.

In [36], the authors use the symmetrical uncertainty to reduce the number of features and improve the performance of the algorithm. It consists in computing correlation between pairs of features based on entropy and mutual information, to keep relevant features and remove redundancy.

Another method for feature selection is based on tree-based 
learning algorithms. These algorithms allow to evaluate the importance of each feature for predicting the target output. Feature importances are by-products of the tree-based algorithm and can be used to identify the most important features, such as in [40]-[43]. This can also give insight about the power system dynamic security assessment problem under consideration.

Feature selection algorithms allow to select the most relevant features. Another field of feature engineering is the feature extraction field. It consists in transforming the data to represent it in a more meaningful way, facilitating the learning. Generally, it also reduces the dimensionality of data. PCA and its variants are often used as a feature extraction tool [44][46], as well as Fisher's linear discriminant [47] and shapelets for time series [48] but recently, an approach based on deep learning auto-encoders was proposed [49]-[52].

Furthermore, feature selection can help to find the most useful PMUs in a network, e.g. the least number and best locations of PMUs for a given DSA application [37], [42].

2) Class imbalance: In most databases, the proportion of stable observations is much larger than the proportion of unstable ones. This is due to the high reliability of power systems. However, this imbalance between classes can degrade the quality of the learnt models, that could be biased toward the majority class. The problem is particularly important, given that unstable events must be detected to guarantee the reliability of the system. To overcome this issue, several solutions have been proposed in the literature, such as oversampling the minority class [24], [53], [54], undersampling the majority class, exploiting a cost-sensitive learning [53], [55] and using ensemble methods [41], [45], [56], [57].

Oversampling is usually preferred to undersampling, to avoid loss of information. The Synthetic Minority Oversampling Technique (SMOTE) adds new observations of the minority class by interpolating linearly data points between adjacent observations of the minority class. A variant of the SMOTE technique is used in [53], combined with costsensitive learning to compensate for class imbalance. Costsensitive learning consists in using different costs for different classes during learning, in order to, for instance, avoid as much as possible misclassifying unstable samples. Using another approach, suitable because of their data representation as images, Hou et al. artificially increase the number of samples of the minority class by using a multi-window sliding recognition method [54]. The database generation method proposed by Venzke et al. in [24] allows to generate balanced dataset between secure and insecure labels.

Ensemble methods can also help for class imbalance problems. For instance, in [56] the authors exploit the diverse extreme learning machine algorithm to determine transient stability. They show that adding diversity can improve accuracy in case of the class imbalance problem. Another example is the use of bagging and, instead of sampling uniformly in the training set, sampling to obtain balanced subsets of training set [45]. Finally, it is possible to use an Adaboost ensemble method, which adapts itself to class imbalance [57], or a 'Weighted Random Forest', that gives more weight to the unstable observations when learning [41].
Reference [55] uses an ad hoc loss to avoid instability detection while [53] uses distinct costs for stable and unstable observations while learning, in order to minimise the misclassification of unstable samples.

\section{Learning a model}

Many learning algorithms have been tested to build models for DSA \& DSC. Most of the time, training is done offline but the model is sometimes updated online. Recently, given the success of deep (neural networks) learning, several papers have proposed to use this technique. However, other learning algorithms such as decision trees and Support Vector Machines (SVMs) are still exploited. Ensemble methods are also proposed in the literature to improve performances. In this section, we present how ML algorithms are exploited for dynamic security by considering first security assessment, then emergency control and finally preventive control.

1) Security assessment : DSA applications are particularly suitable to apply machine learning techniques and many papers exploiting ML to improve DSA were found over the last five years. Table III provides an overview of the main machine learning algorithms used for DSA. The acronyms that appear in the table are defined in the discussion below.

Since many learning algorithms exist, all with their advantages and disadvantages, comparing several algorithms with your dataset is the best way to know which algorithm is more suitable for your application. In [28], a decision tree model is compared to SVMs and neural networks for transient stability assessment. The same algorithms are compared in [58], but this time considering also the random forest algorithm. Reference [85] compares decision trees, SVMs, core vector machines and naive Bayes models while [91] compares several ensemble methods (XGBoost, Bagging, Random Forest, and AdaBoost) with Naive Bayes, $k$ Nearest Neighbor $(k \mathrm{NN})$ and decision trees, this time for voltage stability assessment. In [59], the authors propose an automated multi-model approach for online security assessment. In [60], several learning algorithms such as random forests, Kohonen networks and hybrid neural networks were tested to predict the security status, considering several labels such as normal, alarm, serious alarm and emergency. Note that, depending on the application, the most suitable algorithms may change.

TABLE III

SUPERVISED LEARNING ALGORITHMS USED FOR DSA IN THE LAST FIVE YEARS AND CORRESPONDING REFERENCES

\begin{tabular}{|c|c|c|}
\hline \multicolumn{2}{|c|}{ Algorithms } & References \\
\hline \multirow{4}{*}{ Neural Networks } & FFNN & {$[22],[28],[58]-[69]$} \\
\hline & $\mathrm{CNN}$ & {$[49],[54],[55],[70]-[74]$} \\
\hline & ELM & $\begin{array}{c}{[19],[29]-[33],[35],[56],} \\
{[59],[75]-[80]}\end{array}$ \\
\hline & RNN & {$[37],[81]-[84]$} \\
\hline \multicolumn{2}{|c|}{ Support Vector Machines (SVM) } & $\begin{array}{c}{[15],[28],[39],[50],[51]} \\
{[57]-[59],[85]-[90]}\end{array}$ \\
\hline \multirow{2}{*}{ Tree-based methods } & Decision trees & $\begin{array}{l}{[28],[36],[42],[44],[48]} \\
{[58],[59],[85],[91]-[96]}\end{array}$ \\
\hline & Ensemble & $\begin{array}{c}{[27],[40],[41],[43],} \\
{[58]-[60],[91],[97]-[101]}\end{array}$ \\
\hline \multicolumn{2}{|c|}{$k$ Nearest Neighbor $(k \mathrm{NN})$} & {$[45],[91]$} \\
\hline
\end{tabular}


Recently, due to the success of deep neural networks in other applications, many researchers tackled the dynamic security assessment problem with such an approach. Classical FeedForward Neural Networks (FFNNs) are used as classifiers to predict the small-signal stability [61], oscillatory stability [62] or transient stability [63]; or as regressors to predict the CCT or damping ratio [22], the CCT and final value of rotor angle after a fault [64], the load stability margin [65] or a voltage stability index [66]. In [67], a MapReduce algorithm is used to parallelise the learning of several networks and predict both stability status and the transient stability index. In [68], when the prediction is not credible, time-domain simulations are used to improve the efficiency and in [69], in the context of pre-fault assessment, contingencies are first clustered and then a multi-label neural network is learnt per cluster, to predict the stability status after the occurrence of each contingency in the cluster.

Reference [46] proposes to use deep belief neural networks for voltage stability and another ensemble of neural networks with random weights to predict first if there will be a voltage collapse and then, if no voltage collapse is detected, the transient voltage severity index [26]. Another neural network approach is the Extreme Learning Machine (ELM) algorithm. It is fast to train, and thus can be easily updated during operation. In the literature, ensembles of ELMs are used mostly for stability classification [19], [29], [32], [35], [56], [75], [76], or combined with random vector functional links [31], [33], [77]. It was also used for regression, to predict load stability margins [78], the fault-induced voltage recovery [30], [79], and maximum frequency deviation and time [80].

Convolutional Neural Networks (CNNs) have also been used, in particular for transient stability assessment [49], [54], [55], [70]-[73]. Reference [74] proposes to represent the power system state as a (3-channel) image to take advantage of the convolutional neural network algorithm for small-signal stability. In [70], a twin convolutional SVM network is used while in [72] a hierarchical self-adaptive method, with one CNN per type of features, determines the stability of the system. In [73], a cascade of CNNs works with time-domain simulation to improve efficiency for pre-fault assessment. Time domain simulations are performed one cycle at a time and are used as inputs of the CNN. If the prediction of the CNN is credible, time-domain simulations are stopped.

Recently, Recurrent Neural Networks (RNNs) were also proposed, because of their ability to consider temporal correlations, either with Long Short-Term Memory (LSTM) units [37], [81], [82] or Gated Recurrent Units (GRU) [83], [84].

More classical algorithms are however still exploited in the literature. For instance, SVM methods are used for transient stability [50], [51], [86]-[88] or voltage stability [39], [89]. SVMs are easier to train than neural network models and they can be used to predict the stability status of the power system (stable or unstable) or a voltage stability margin index [89]. The parameters of the SVM can be optimised with a grid search algorithm or particle swarm optimisation [88], [89]. In order to increase the accuracy of the assessment, in [87], two SVM models are used, an aggressive and a conservative one. This allows to predict a third class, called the grey region,

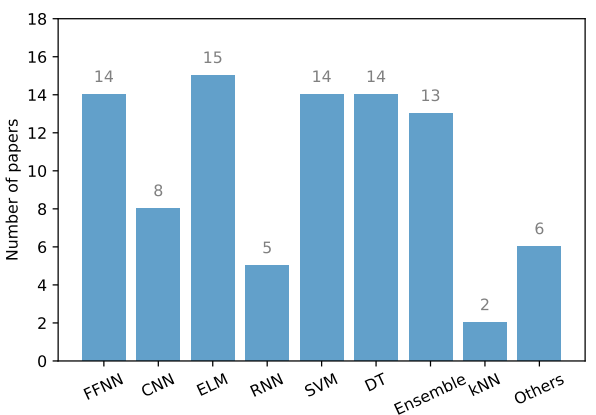

Fig. 6. Number of papers addressing power system dynamic security assessment problems per machine learning algorithms

when both models disagree, to indicate when the assessment is uncertain. Ensembles of SVMs [57], [90] or variants of the SVM such as core vector machines [15] have also been proposed to increase the accuracy of the classification.

Tree-based methods are still popular in the power system literature. A huge advantage of them is their interpretability, which is important in reliability management. Single Decision Trees (DTs) are used to predict the stability status of the system [36], [42], [44], [48], [92]-[94]. In [94], the authors explore a trade-off between predictive accuracy and interpretability. To improve accuracy, ensembles of decision trees such as Adaboost [27], [97], XGboost [43] and Random Forests [40], [41], [98]-[101] are proposed. In [101], uncertain predictions are checked with time-domain simulations. In the European project iTesla, decision trees are used for online security assessment. The platform developed within this project for online static and dynamic security assessment is presented in [95], [96].

The simple $k \mathrm{NN}$ algorithm is used in [45] with bagging to predict transient stability. Logistic regression, after a stacked denoising auto-encoder is used in [52] to predict post-fault stability status. Less common approaches have also been proposed, such as unsupervised learning with PCA [102] and semi-supervised learning when few observations are labelled [19]. In the latter, the 'Tri-training' algorithm is used, which consists in training three models with a small subset of labelled data and then adding an unlabelled sample to the training set of a classifier only if the two other classifiers agree.

Figure 6 summarises the number of papers per learning algorithm for DSA. One can notice that neural network algorithms have been very popular in the last five years.

2) Emergency control: Instead of predicting the stability state of the system, data-driven models can be used to choose an emergency control decision, or to give insight about the best corrective control actions for the actual situation. Table IV provides an overview of the publications about dynamic security emergency control according to the main machine learning approach used.

Reinforcement learning [16], [103], [104] or adaptive dynamic programming [105], [106] are used to improve voltage, frequency or transient stability. Supervised learning techniques can also be used. For instance, in [47], the authors train a decision tree classifier to evaluate transient stability and thanks to the Fisher linear discriminant, they evaluate the sensitivity of each generator and load to stability and then 
TABLE IV

ML PROTOCOLS AND SL ALGORITHMS USED FOR DYNAMIC SECURITY EMERGENCY CONTROL OVER THE LAST FIVE YEARS

\begin{tabular}{|c|c|}
\hline Methods & References \\
\hline Reinforcement learning & {$[16],[103],[104]$} \\
\hline Adaptive programming & {$[105],[106]$} \\
\hline Unsupervised learning & {$[107]$} \\
\hline Tree-based algorithms & {$[47],[108]-[110]$} \\
\hline Neural networks & {$[111]-[113]$} \\
\hline
\end{tabular}

define emergency control actions accordingly. In [111], the authors use a neural network to assess the generators that need to be re-dispatched and the loads that need to be shed.

In [107], patterns of unstable dynamic behaviour in the dataset are identified with an unsupervised learning technique and then a classifier is used to determine in which pattern the actual situation falls. This indicates which generators may lose synchronism to help for emergency control decisions.

In [108], an ensemble of decision trees is used to assess and control voltage stability. There is one decision tree per possible combination of control action status ( 1 or 0 , depending if they are used or not), indicating which control combinations lead to a secure system. The control actions combination chosen is the one leading to a secure action with a minimum number of control devices used. This approach is also used in [109], but there is first identification of voltage control areas, to reduce control candidates.

In [112], the authors propose to control a hybrid energy storage system for load-frequency control. They design an adaptive control based on a neural network, the design of which being facilitated by a Hammerstein-type neural network to identify the storage system. In [110], the authors use a proximity driven streaming random forest algorithm with L-index as indices for voltage stability. The algorithm can determine corrective and/or preventive control actions, such as additional reactive power injections, and localise critical nodes, where the system is close to a stability loss.

In the context of transient stability, in [113] a neural network is used to estimate the gain for a static synchronous compensator auxiliary controller, that needs to be adjusted according to the system operation point to obtain the desired CCT. The gain computation is heavy and thus a neural network model is proposed to reduce the computational burden.

3) Preventive control: Several approaches have been proposed in the literature for preventive control regarding dynamic security. Table $\mathrm{V}$ provides an overview of the publications about dynamic security preventive control according to the main machine learning approach used.

The first approach consists in using machine learning to predict the preventive control scheme. For instance, in [114], the authors propose to use multi-objective reinforcement learning for short-term voltage stability, in order to minimise both voltage deviation and control action cost.

The second approach consists in identifying candidate control actions for preventive control. In [115], the authors propose a preventive control scheme by rescheduling generating units. First, they assess the transient stability of the system
TABLE $\mathrm{V}$

ML PROTOCOLS AND SL ALGORITHMS USED FOR DYNAMIC SECURITY PREVENTIVE CONTROL OVER THE LAST FIVE YEARS

\begin{tabular}{|c|c|}
\hline Methods & References \\
\hline Reinforcement learning & {$[114]$} \\
SVM & {$[34],[38],[115]$} \\
\hline Neural networks & {$[116]$} \\
Tree-based methods & {$[117]-[121]$} \\
\hline Linear models & {$[122]$} \\
\hline
\end{tabular}

with a hybrid method based on a SVM model and time-domain simulation. Then, if the system is unstable, they compute from the SVM model the sensitivity of each generator to a transient stability assessment index (derived from the SVM model) to rank the generators and select the ones that are more effective for improving the stability. In [38] the authors exploit SVMs for determining the coherency of generating machines in the context of transient stability. The purpose is to rank generators according to their vulnerability, based on a transient stability index, to facilitate preventive control (rescheduling of generation). Finally, in [32], the authors use the Relief-F feature selection method to identify critical generators modifying the security status of the operating condition. These generators are then considered as candidate control variables for preventive control. A bit different, but still to help preventive control, Mokhayeri et al. propose a method based on decision trees for detecting the apparition of islands [117].

Another main approach, quite recent, consists in building models of dynamic security assessment and then extracting security rules from these models that can be embedded in optimisation problems, to define control actions considering dynamic security. Indeed, classifiers built with machine learning contain knowledge about stable and unstable regions. Cremer et al. exploit decision trees to embed the rules determining the output of the classifier in a decision-making problem (i.e. an OPF) [118]. This allows to compute control decisions considering the stability boundary. In their paper, the authors present the challenges of this approach, which are the computational complexity to build the database and the accuracy of the such sample-derived rules. This approach is further developed in [119], where learnt condition-specific safety margins are proposed to be incorporated in a decision-making program. These margins allow, according to the authors, to improve the risk/cost balance. An ensemble of decision trees (Adaboost) is used to perform probabilistic security control.

In [120], the authors propose to build with machine learning line flow constraints to be incorporated in a market clearing program (under the form of a SCOPF) to improve both smallsignal stability and steady-state security. They use a decision tree-based classifier to extract knowledge. The decision trees rules consist in conditional line transfer limits, that can be embedded in the SCOPF in order for the operator to take decisions already in line with the small-signal stability margin. An extension of this work to solve an AC-SCOPF instead of a DC-SCOPF is proposed in [121], while still incorporating N1 security and small-signal stability with decision tree-learnt rules. In [116], the authors are solving an OPF considering 
transient stability constraints. An artificial neural network approximating the CCT of a fault is embedded in the OPF formulation. This guarantees that the preventive decisions will be such that the CCT of all considered faults is greater than a defined minimum value. A bit different but still embedding a machine learnt model in an optimisation program, the authors of [34] build a two-stage SVM model to determine the transient stability region that is embedded in a decisionmaking program to determine preventive control actions. The final transient stability-constrained OPF being non linear and non convex, the authors propose to solve it with particle swarm optimisation. Finally, in [122], the authors propose to automatically learn operating rules for a stability constrained system.

\section{Validating and maintaining a machine learnt model}

In this section, we present first how researchers in reliability management proposed to deal with the frequent system changes in a power system and then what can be done when data is missing or erroneous.

1) Dealing with system changes: System changes such as topology changes are common in power systems but they can impact the quality of prediction of the machine learnt models. To overcome this issue, several papers propose to regularly retrain or update the model with new data acquisition. Regarding neural network models, in [65], the model is updated with misclassified samples when a certain number of errors occurred. Online sequential ELM models were also proposed, as in [32], [80], because they are fast to train and can be updated regularly. Another approach is to use a recurrent neural network. In [83], an online monitoring system, consisting in a stacked GRU based recurrent neural network, is shown to be able to adapt to topology change. Concerning treebased methods, Yang et al. update decision trees in real-time using an online boosting method [108], while [97] presents a very fast decision tree system based on Hoeffding trees to quickly update online an Adaboost ensemble of decision trees. Furthermore, Tomin et al. propose the proximity driven streaming random forest algorithm that can independently and adaptively change the model [110].

Another solution proposed in the literature is active learning [71], [123]. For instance, in [123], the authors propose an active learning solution that consists in updating the model with real samples when the model prediction is not consistent with the actual system condition. More specifically, they train and update the model with data for which the prediction contradicts with the actual stability state of the system.

2) Missing or erroneous data: During online assessment or control, when exploiting PMU data, many events can occur such as PMU malfunctioning, time delays, communication loss, noise in the measurements and loss of data packets. Therefore, it is important to develop models able to deal with these missing data or erroneous measures. When erroneous measure are outliers, they can be detected, for example by using the Z-score algorithm [65]. Concerning missing data or detected erroneous measures, it is possible to estimate their values, either by interpolation techniques such as polynomial curve fitting technique [65] or by using machine learning techniques such as an ensemble of extreme learning machines and random vector functional links [33] or the emerging deep learning technique called Generative Adversarial Networks (GAN) [77]. Instead of replacing missing data with estimations, some authors try to build a model robust to incomplete measurements by extending the training database with samples containing incomplete measurements [86].

\section{RECENT WORKS IN SSA \& SSC}

The methodology to exploit machine learning for static security assessment and control is similar to the one used for dynamic security, although the input variables and target outputs vary in function of the application. Therefore, we directly present how ML is applied to solve the different static security assessment and control problems. We organised this section considering the power system tool that is considered to be replaced or enhanced with machine learning techniques. In particular, we consider power flow computation, optimal power flow solving, and unit commitment optimisation.

\section{A. Prediction of power flows}

Table VI sets out the main target outputs of the ML methods used in the context of static security assessment, to replace or enhance power flow computations, the exploited learning algorithms, as well as their corresponding references.

Some papers studied the possibility to replace the power flow computation by a proxy, for a faster static security assessment. For instance, [124] uses a deep neural network to estimate power flows very quickly. The authors propose to exploit it to help operators in the control room to choose remedial actions such as network topology modification after a contingency. Improving their previous work, they introduced guided dropout to enable the estimation of flows for a range of power system topologies [125]. The guided dropout method uses some neurons that are only activated if the corresponding

TABLE VI

MAIN ML APPROACHES FOR POWER FLOWS PROBLEMS OVER THE LAST FIVE YEARS AND CORRESPONDING REFERENCES

\begin{tabular}{|c|c|c|}
\hline Predicted quantities & Algorithms & References \\
\hline \multirow{2}{*}{$\begin{array}{c}\text { Power } \\
\text { flows }\end{array}$} & Neural networks & [124]-[129] \\
\hline & SVM & [130] \\
\hline \multirow{2}{*}{$\begin{array}{c}\text { (Composite) } \\
\text { Security indices }\end{array}$} & Neural networks & [131]-[133] \\
\hline & LASSO & [134] \\
\hline \multirow{2}{*}{$\begin{array}{l}\text { Security } \\
\text { status }\end{array}$} & Tree-based methods & {$[135]-[140]$} \\
\hline & SVM & {$[141],[142]$} \\
\hline \multirow{3}{*}{$\begin{array}{l}\text { Security } \\
\text { margin }\end{array}$} & Neural networks & {$[143],[144]$} \\
\hline & SVM & {$[145]$} \\
\hline & Tree-based methods & [146] \\
\hline \multirow{3}{*}{$\begin{array}{c}\text { Critical } \\
\text { areas }\end{array}$} & Unsupervised learning & {$[18],[147]$} \\
\hline & Neural networks & [148] \\
\hline & SVM & [149] \\
\hline $\begin{array}{c}\text { Transmission } \\
\text { reliability margin }\end{array}$ & Neural networks & {$[150]$} \\
\hline $\begin{array}{c}\text { Total } \\
\text { transfer capability }\end{array}$ & Neural networks & {$[151]$} \\
\hline
\end{tabular}


line/breaker is disconnected, and the authors show that with this approach, a proxy trained with only $\mathrm{N}-1$ events can generalise to $\mathrm{N}-2$ events. This proxy is fast, and can be used to rank (double) contingencies and estimate the risk of a grid state [126], [127]. Finally, using historical data, a new type of neural networks for predicting line flows in case of topology change is presented in [128], where the authors introduce the Latent Encoding of Atypical Perturbation (LEAP net) architecture, so as to further improve the generalisation to unseen topologies.

Another proposal to predict line flows is to use SVMs with linear kernels [130]. The proxy of power flows is used in the context of designing a reserve policy, for better reserve scheduling and allocation. In [129], a proxy of load flow computation is built with an adaptive neuro-fuzzy inference system to predict the voltage magnitude at all buses.

Load flow computations are also used to evaluate security indices, indicating violation of transmission constraints, or to evaluate composite security indices, indicating violation of both bus voltage and power line transmission constraints. Computing these indices can help the operator to rank the considered contingencies according to their severity. In [131], an artificial neural network is used to predict a security index for each considered contingency. In [132], Radial Basis Function neural networks are used to predict the composite security index directly without computing load flows for each contingency, in order to rank the contingencies. Features are selected based on a Single Ranking and Correlation Coefficient approach. In [133], the authors propose a neural network trained with PSO, while, in [134], they use a LASSO (Least Absolute Shrinkage and Selection Operator) method.

On the other hand, some papers assess the static security of the system with security labels. For instance [135] uses decision trees and random forests for static security assessment. [139] uses a deep auto-encoder to extract features and proposes an objective-based loss function to learn the auto-encoder, in such a way that it minimises the misclassification of unstable observations. Multiway decision trees were exploited in [140] to determine if the system is secure or insecure while considering system topology. Furthermore, Stratified Random Sampling was used to obtain the same proportion of secure and insecure labels. In [141], the authors exploit the SVM approach to classify contingencies as secure, alarm or insecure and in [136], the authors test four decision tree algorithms to classify the N-1 contingencies as secure or insecure. Both papers also apply a sequential search algorithm for feature selection. Finally, in [142] the authors propose several Adaboost classifiers with SVMs as weak learners to evaluate the static security state of the system and then evaluate in case of insecure state the type of condition violation. Feature selection is performed based on class separability and correlation coefficient indices, while the class imbalance is corrected using a SMOTE method.

In the context of static voltage stability assessment, several load flows may be run to determine the voltage collapse point and thus the voltage stability margin. In this context, Fan et al. [152] propose a feature selection method based on the maximum relevance minimum redundancy algorithm. The authors repeat this procedure several times, starting with a different feature each time, to obtain an ensemble of different subsets of features. Then they perform curve fitting for each subset in order to infer a relation between the features and the voltage stability margin and they average the results to obtain one prediction. In [137], the authors use PMU data for online voltage stability assessment and deal with the problem of frequent model update. Frequently updating the learning model is useful to take system changes into account but it can take time. To tackle this issue, the authors propose a random forest algorithm, where only part of the decision trees are updated each time instead of the whole model. They use a weighted majority vote for the final prediction. They also perform variable selection based on variable importances, a by-product of random forest algorithms. Reference [145] exploits the SVM learning algorithm (with a GA to optimise the parameters of the SVM) to estimate the stability margin for a given contingency. The final purpose is to estimate the static voltage security risk. Reference [143] uses a neural network to predict a unified voltage stability indicator and [144] exploits a Radial Basis Function network to estimate a probabilistic insecurity index. Finally, in [146], the authors compare two feature selection methods, Relief-f and participation factor, to predict the static voltage security margin with a decision tree. They show that both methods are equivalent.

In [138], a hybrid approach of random forests and tree boosting is used for the steady-state security analysis. They consider four classes, normal, alarm, emergency correctable and emergency non correctable.

Machine learning approaches can also be used for detection of critical areas. In [148], deep learning is used for the detection of security weak spots using spatio-temporal and meteorological data. In [147], the authors exploit the unsupervised learning algorithm called k-means to identify voltage stability critical areas, while in [149] SVMs are used to detect these weak areas and then assess the voltage stability of each area. Reference [18] proposes to exploit an unsupervised learning technique to segment a power system into zones, helping the operators managing the grid.

Finally, to help security assessment, [150] uses neural networks to estimate the transmission reliability margin. On the other hand, [151] proposes a method to predict total transfer capability (available amount of power that can be transferred on the tie-lines), which is an ensemble model with adaptive hierarchical GA-based neural networks. A hybrid feature selection based on Maximal Information Coefficient (MIC) and nonparametric independence screening is used. The maximal information method is used to reduce redundancy between features by computing the MIC between two features and eliminating one of them if the MIC value is greater than a given threshold. Then the nonparametric independence screening evaluates the correlation between each resting feature and the target output, to keep the ones with the highest correlation.

\section{B. Prediction of optimal power flow features and outcomes}

A more recent application scope of machine learning for reliability management is to help out in solving OPF problems. OPF is extensively used by the operators for operation 
TABLE VII

MAIN ML APPROACHES TO SOLVE OPF PROBLEMS OVER THE LAST FIVE YEARS AND CORRESPONDING REFERENCES

\begin{tabular}{|c|c|c|}
\hline Predicted quantities & Algorithms & References \\
\hline \multirow{2}{*}{ Decisions } & Neural networks & {$[153]-[155]$} \\
\cline { 2 - 3 } & Tree-based methods & {$[156],[157]$} \\
\hline $\begin{array}{c}\text { Related } \\
\text { costs }\end{array}$ & $\begin{array}{c}\text { Several } \\
\text { algorithms }\end{array}$ & {$[158]-[160]$} \\
\hline $\begin{array}{c}\text { LOL } \\
\text { status }\end{array}$ & Neural networks & {$[161],[162]$} \\
\hline \multirow{2}{*}{$\begin{array}{c}\text { Features } \\
\text { of the OPF }\end{array}$} & Neural networks & {$[163],[164]$} \\
\cline { 2 - 3 } & Statistical learning & {$[165],[166]$} \\
\hline
\end{tabular}

planning, to find optimal decisions considering physical and operational constraints. They are also solved repeatedly during operation. However these problems are non linear and non convex and generally large-scale. Furthermore, close to realtime, they must be solved within a short period of time, considering the uncertainties (e.g. demand and renewable generation). Solving OPF problems is thus still a computational challenge, that has been recently addressed with the help of machine learning techniques. In the literature, some papers approach this problem by directly predicting the decisions or related costs of an OPF while others try to learn features of the OPF, in order to enhance its solving. To summarise the different approaches and the exploited learning algorithms, the references discussed below are sorted in Table VII.

Most papers predicting directly the outputs of an OPF predict the decisions given by the program, which are usually generator setpoints (generator active power and voltage magnitude). For instance, in [156], the authors predict the generation dispatch, using random forest models. More recently, several papers proposed to exploit Deep Learning (DL) for this purpose. Pan et al. [153] use deep learning to predict the decision of a DC-OPF while in [154], [155] the authors predict the decision of an AC-OPF. In all these papers a post-processing method ensuring the feasibility of the solution is described. In [153], if the predicted solution is not feasible, the authors solve an optimisation problem to find the feasible solution closest to the predicted one. In [155], the output of the DL model is constrained with a sigmoid function to adhere to active power generation and voltage magnitude constraints and a power flow problem is then solved based on the predictions. Finally, in [154], the authors take advantage of previous optimal power flow solutions computed at previous time steps as well as a dual Lagrangian method to improve the solution and enforce physical and operational constraints.

Rather than predicting the decisions obtained from an OPF, some papers [158]-[160] are interested in the (optimal) cost of operation related to the decisions. In these three papers, several learning algorithms are tested and compared to predict the cost of real-time operation obtained from an AC-OPF [158] and a DC-SCOPF with N-1 security contraints [159], [160].

Another application of OPF is determining if the demand could be met at all buses for given operating conditions. Coupled with Monte-Carlo simulations to generate many op- erating states, it can help assess reliability of the system with reliability indices such as Loss Of Load Probability (LOLP). In [161], [162], the authors use an RBF neural network to predict if the demand is met at all buses. This allows them to estimate LOLP indices without solving an OPF problem for each state, accelerating the reliability assessment of the system.

With a different approach, Baker proposes to learn the outputs of an AC-OPF problem with a random forest but, instead of directly using the predictions of the learnt model, she proposes to exploit the predictions as a warm-start point of the AC-OPF [157]. The author shows on some test systems that it leads to a faster convergence time compared to other warm-start methods.

To reduce the computational burden related to the OPF solving, some papers [157], [163]-[167] propose to exploit machine learning to reduce the search space of the OPF problem and thus accelerate the resolution. In [165] and [166], the authors propose a method to solve a stochastic DCOPF, considering uncertainties. This DC-OPF must be solved within a short time period and one proposed approach is to pre-define an ensemble control policy, before the realisation of uncertainties. Then, in real-time, one has to find with exhaustive search the optimal control policy for the realisation of uncertainties, instead of solving the full OPF in real-time. To define the ensemble control policy, one can search for all the bases (i.e. the sets of active constraints, which are all satisfied with equality for a given solution) of the DC-OPF, given the distribution of uncertainties, and then to each set associate an affine policy. When a scenario is realised, one can look for the set of active constraints corresponding to this scenario and then apply the corresponding affine policy. In order to find an ensemble control policy more efficiently, [165] proposes to leverage statistical learning to identify the most important sets of active constraints of the real-time DCOPF, given the distribution of uncertainties; the most important sets being the ones with a higher probability to be optimal for the OPF. By computing affine control policies for only a subset of bases, the authors reduce the computational burden in real-time, allowing to solve these parametric programs more efficiently online.

Following these works, Deka et al. [164] exploit neural networks to learn a mapping between realised uncertainties and the corresponding optimal set of active constraints, allowing to enhance even more the computational efficiency of the approach. The problem is tackled differently by Ardakani and Bouffard in [163]. Instead of predicting the set of active constraints, they propose to predict with an artificial neural network the set of umbrella constraints, which corresponds to the set with the minimum number of constraints such that if one constraint is removed, the set of feasible solutions of the original OPF problem is modified. Solving the OPF with only the umbrella set of constraints may reduce significantly the solution time of the OPF. Finally, Mezghani et al. propose a different approach to deal with short-term uncertainties when solving OPF. They use the scenario-based approach, where power flows equations are solved for a number of scenarios sampled from the distribution of uncertainties and 
TABLE VIII

MAIN ML APPROACHES TO SOLVE UNIT COMMITMENT (UC) PROBLEMS OVER THE LAST FIVE YEARS AND CORRESPONDING REFERENCES

\begin{tabular}{|c|c|c|}
\hline Predicted quantities & Algorithms & References \\
\hline \multirow{2}{*}{ Decisions } & $k \mathrm{NN}$ & {$[168],[169]$} \\
\cline { 2 - 3 } & $\begin{array}{c}\text { Reinforcement } \\
\text { learning }\end{array}$ & {$[170]-[174]$} \\
\hline $\begin{array}{c}\text { Features } \\
\text { of the UC }\end{array}$ & $k \mathrm{NN}$ & {$[175]$} \\
\hline
\end{tabular}

they propose to exploit machine learning to reduce the number of scenarios needed to solve the problem while keeping an accurate uncertainty quantification [167].

\section{Unit commitment prediction}

The unit commitment problem consists in deciding in advance which generating units should be on or off for the time horizon considered. Accelerating the solution of a unit commitment problem is useful, especially in applications for which a large number of unit commitment problems must be solved. In order to summarise this section, Table VIII presents the ML approaches used for solving the unit commitment problem as well as the corresponding references.

For instance, in [168], the authors need to quickly evaluate the outcome of short-term operation, for a long-term planning purpose. They propose to use $k \mathrm{NN}$ as a proxy of short-term operation and thus the predicted unit commitment schedule is the schedule in the learning database with operating conditions closest to the one evaluated. This proxy can be exploited for maintenance scheduling, by using short-term operation proxies to quickly evaluate the impact of a maintenance decision on power system operation [169]. Managing the grid at different time scales is a complex task. To reduce the computational burden when considering different time scales for decisionmaking (for instance day-ahead and real-time), Dalal et al. exploit the reinforcement learning approach [170].

The approach for solving OPF can also be applied for unit commitment problems. For instance, in [175], the authors propose to reduce the dimensional complexity of a transmissionconstrained unit commitment problem by learning the congestion status of transmission lines and disregarding lines that will not become congested; i.e. removing redundant or inactive constraints. The learning algorithm chosen is also the $k \mathrm{NN}$.

Finally, the unit commitment problem can also be addressed with a reinforcement learning approach. In [171], the authors exploit reinforcement learning to choose a unit commitment and economic dispatch in a hierarchical way, while minimising operation cost or $\mathrm{CO}_{2}$ emissions; and in [172] the unit commitment problem is modelled as a Markov decision process. The unit commitment problem can also be defined as a multiobjective problem, for example to both minimise operating costs and maximise system reliability [173] or minimise both operation costs and wind curtailment [174]. To solve this difficult problem, both papers exploit reinforcement learning coupled with particle swarm optimisation.

\section{OTHER RELATED APPLICATIONS}

In this section, we want to shortly point to three other areas of application where machine learning has been proposed as a tool, and which are related to power system reliability management. For each category, we briefly explain the nature of the application and its relation with reliability management, and then point to some recent survey/review or tutorial publications discussing machine learning in the context of the topic.

Fault detection, classification and localisation applications consist respectively in detecting, identifying and localising faults or disturbances in a power system. These methods can help to improve significantly the reliability of power systems by allowing faster and/or more selective reactions in the context of protections, emergency and corrective control. Over the years, several machine learning approaches have been proposed in the scientific literature to address these problems, and we refer the interested reader to [176], [177] for an overview of recent literature on this subject.

Power system operation relies heavily on the availability in the control centre of an accurate and timely characterisation of the system state. State estimation refers to a set of methods and algorithms exploiting real measurements and event recordings to build up such a characterisation in real-time, in a way that is robust to noise, gross errors, modelling uncertainty and telecommunication delays and errors. The resolution of this problem can be enhanced by leveraging machine learning. In particular, in the context of active distribution systems management this may be useful, since in this area state estimation is not yet a well established technology. We refer the reader to [178], [179] for further information about ongoing works in these directions.

Finally, load or renewable energy production forecasting is an extremely important and multifaced class of problems that becomes more and more important for suitable decision making in operation and operation planning of electric power systems. Many ML methods have been proposed to improve the quality and the scope of the point forecasts of individual quantities, to model spatio-temporal dependencies, and to provide ensemble forecasts [180]-[182].

\section{DISCUSSION}

In this paper, we reviewed recent works tackling electric power systems reliability assessment and control problems with various machine learning techniques. Indeed, a large increase in the number of publications in that particular field was observed recently. Although most papers deal with reliability assessment, more and more papers propose new applications for reliability control, such as solving OPF problems with the help of supervised learning or using reinforcement learning for the design of closed loop control schemes.

However, despite the great potential of these techniques, some challenges still must be faced before machine learning becomes common practice for reliability assessment and control in the electric power system industry. We detail hereafter some challenges that we believe should be addressed by further research and development activities. 
A first challenge concerns the acceptance of these new methods by the human users (operators and planners) in the industry. The traditional approaches used in practice for reliability management are model-based and take explicitly into account well-known physical laws. Given the practical consequences of a failure in assessment or control, moving towards a data-driven approach is difficult. There is definitely a need to convince the field experts that these methods are actually efficient and reliable. First, approaches using machine learning should be used in parallel with the more traditional approaches, to allow the human experts to assess their accuracy and usability; subsequently both approaches could be used in symbiosis, where traditional approaches would only be applied when the machine learnt predictors are not confident enough in their predictions. Furthermore, we believe that anyhow interpretability of the machine-learnt models is something that could not be neglected in future research. Another challenge comes with the fact that electric power systems are constantly changing. Therefore, ensuring the adaptability and proposing ways to maintain over time the quality of the machine-learnt models used for assessment and control is also a requirement for the practical acceptance of machine learning applications to reliability management of electric power systems.

With the rise of data-driven methods, vulnerability of machine learnt models against man-crafted adversarial data must also be considered [183] and techniques to detect these adversarial examples should be developed. More broadly, an important aspect not to be neglected is the study of guarantees on the performances of machine learning algorithms, in particular to avoid unexpected or harmful behaviour [184]. This is necessary for system operators to trust new proposals of machine learning for reliability management.

Beyond these current practical challenges discussed hereabove, we also see many interesting future research directions in the field of machine learning for reliability management of large scale systems of systems such as electric power systems.

As a first direction for future research, we believe that reliability databases and evaluation protocols should be built and made publicly available. It would allow to more easily compare the various methods proposed in the literature and would help researchers in that field to advance more rapidly. Similarly, data shared by the industrial system operators would also help the research community, but other types of problems such as privacy, safety, and commercial sensitivity, are major obstacles for letting this happen in a near future.

Another direction of research, quite new, that we expect to be more developed in a recent future, is the use of machine learnt models, that are called proxies, to model the behaviour of other parts of the overall multi-energy system. These can be smaller subsystems or other large-scale systems interacting with the managed one, such as distribution grids, other interconnected transmission systems, gas transportation systems, electric vehicle charging infrastructures, district heating systems, etc. One can also consider the integration of different time scales for sequential decision-making, and then use for instance proxies modelling the real-time operation in order to enhance the decision making process in short-term operation planning, or proxies of post-contingency behaviour of emergency control systems to be exploited in the context of preventive mode dynamic security assessment.

To conclude, we think that the proposed methods are of great potential to improve reliability assessment and control and we expect more and more applications, both in research and in industry, to be developed while exploiting machine learning techniques. Finally, we believe that the methods mentioned and the identified challenges and future directions of research are relevant for many other large scale systems and infrastructures, even beyond energy systems, such as distribution grids, micro-grids and multi-energy systems.

\section{REFERENCES}

[1] L. A. Wehenkel, Automatic learning techniques in power systems. Springer Science \& Business Media, 2012.

[2] L. H. Fink and K. Carlsen, "Operating under stress and strain [electrical power systems control under emergency conditions]," IEEE Spectrum, vol. 15, no. 3, pp. 48-53, March 1978.

[3] R. Billinton and R. N. Allan, Reliability evaluation of power systems, 2nd ed. New York: Plenum, 1996

[4] E. Litvinov and F. Zhao, "Survivability of the electric grid," in Bulk Power System Dynamics and Control (iREP) - X (iREP), 2017 iREP Symposium, 2017.

[5] T. E. Dy Liacco, "The adaptive reliability control system," IEEE Transactions on Power Apparatus and Systems, no. 5, pp. 517-531, 1967.

[6] P. Kundur, J. Paserba, V. Ajjarapu, G. Andersson, A. Bose, C. Canizares, N. Hatziargyriou, D. Hill, A. Stankovic, C. Taylor, T. Van Cutsem, and V. Vittal, "Definition and classification of power system stability IEEE/CIGRE joint task force on stability terms and definitions," IEEE Transactions on Power Systems, vol. 19, no. 3, pp. 1387-1401, Aug 2004.

[7] P. Kundur, N. Balu, and M. Lauby, Power System Stability and Control, ser. EPRI power system engineering series. McGraw-Hill Education, 1994. [Online]. Available: https://books.google.be/books? id=2cbvyf 8 Ly 4 AC

[8] GARPUR Consortium, "Guidelines for implementing the new reliability assessment and optimization methodology," 7th framework programme, EU Commission grant agreement 608540, Nov. 2016. [Online]. Available: https://www.sintef.no/projectweb/garpur/deliverables/

[9] F. Capitanescu, J. Martinez Ramos, P. Panciatici, D. Kirschen, A. Marano Marcolini, L. Platbrood, and L. Wehenkel, "State-of-theart, challenges, and future trends in security constrained optimal power flow," Electric Power Systems Research, vol. 81, no. 8, pp. 1731-1741, 2011.

[10] B. Stott and O. Alsac, "Optimal power flow - basic requirements for real-life problems and their solutions," Jan. 2012.

[11] F. Capitanescu, "Critical review of recent advances and further developments needed in AC optimal power flow," Electric Power Systems Research, vol. 136, pp. 57 - 68, 2016.

[12] J. Friedman, T. Hastie, and R. Tibshirani, The elements of statistical learning. Springer series in statistics New York, 2001, vol. 1, no. 10

[13] K. P. Murphy, Machine learning: a probabilistic perspective. MIT press, 2012.

[14] R. S. Sutton and A. G. Barto, Reinforcement learning: An introduction. MIT press, 2018.

[15] B. Wang, B. Fang, Y. Wang, H. Liu, and Y. Liu, "Power system transient stability assessment based on big data and the core vector machine," IEEE Transactions on Smart Grid, vol. 7, no. 5, pp. 2561$2570,2016$.

[16] R. Yousefian, R. Bhattarai, and S. Kamalasadan, "Transient stability enhancement of power grid with integrated wide area control of wind farms and synchronous generators," IEEE Transactions on Power Systems, vol. 32, no. 6, pp. 4818-4831, 2017.

[17] M. Glavic, R. Fonteneau, and D. Ernst, "Reinforcement learning for electric power system decision and control : Past considerations and perspectives," IFAC-PapersOnLine, 2017. [Online]. Available: https://www.sciencedirect.com/science/article/pii/S2405896317317238 
[18] A. Marot, S. Tazi, B. Donnot, and P. Panciatici, "Guided machine learning for power grid segmentation," in 2018 IEEE PES Innovative Smart Grid Technologies Conference Europe (ISGT-Europe). IEEE, 2018, pp. 1-6.

[19] R. Liu, G. Verbič, and J. Ma, "A new dynamic security assessment framework based on semi-supervised learning and data editing," Electric Power Systems Research, vol. 172, pp. 221-229, 2019.

[20] I. Guyon and A. Elisseeff, "An introduction to variable and feature selection," Journal of machine learning research, vol. 3, no. Mar, pp. $1157-1182,2003$.

[21] F. Thams, A. Venzke, R. Eriksson, and S. Chatzivasileiadis, "Efficient database generation for data-driven security assessment of power systems," IEEE Transactions on Power Systems, pp. 1-12, 2019.

[22] S. Jafarzadeh and V. M. I. Genç, "Probabilistic dynamic security assessment of large power systems using machine learning algorithms," Turkish Journal of Electrical Engineering and Computer Sciences, vol. 26, no. 3, pp. 1479-1490, 2018.

[23] I. Konstantelos, M. Sun, S. H. Tindemans, S. Issad, P. Panciatici, and G. Strbac, "Using vine copulas to generate representative system states for machine learning," IEEE Transactions on Power Systems, vol. 34, no. 1, pp. 225-235, 2019.

[24] A. Venzke, D. K. Molzahn, and S. Chatzivasileiadis, "Efficient creation of datasets for data-driven power system applications," Arxiv, pp. 1-8, 2019. [Online]. Available: http://arxiv.org/abs/1910.01794

[25] T. E. Huang, Q. Guo, and H. Sun, "A distributed computing platform supporting power system security knowledge discovery based on online simulation," IEEE Transactions on Smart Grid, vol. 8, no. 3, pp. 15131524, 2017.

[26] Y. Xu, R. Zhang, J. Zhao, Z. Y. Dong, D. Wang, H. Yang, and K. P. Wong, "Assessing short-term voltage stability of electric power systems by a hierarchical intelligent system," IEEE Transactions on Neural Networks and Learning Systems, vol. 27, no. 8, pp. 1686-1696, 2015.

[27] F. Hang, S. Huang, Y. Chen, and S. Mei, "Power system transient stability assessment based on dimension reduction and cost-sensitive ensemble learning," 2017 IEEE Conference on Energy Internet and Energy System Integration (EI2), 2017.

[28] N. G. Baltas, P. Mazidi, J. Ma, F. de Asis Fernandez, and P. Rodriguez, "A comparative analysis of decision trees, support vector machines and artificial neural networks for on-line transient stability assessment," in 2018 International Conference on Smart Energy Systems and Technologies (SEST). IEEE, 2018, pp. 1-6.

[29] Y. Zhang, Y. Xu, Z. Y. Dong, Z. Xu, and K. P. Wong, "Intelligent early warning of power system dynamic insecurity risk: Toward optimal accuracy-earliness tradeoff," IEEE Transactions on Industrial Informatics, vol. 13, no. 5, pp. 2544-2554, 2017.

[30] Y. Zhang, Y. Xu, Z. Y. Dong, and R. Zhang, "A hierarchical selfadaptive data-analytics method for real-time power system shortterm voltage stability assessment," IEEE Transactions on Industrial Informatics, vol. 15, no. 1, pp. 74-84, 2018.

[31] C. Ren, Y. Xu, Y. Zhang, and C. Hu, "A multiple randomized learning based ensemble model for power system dynamic security assessment," IEEE Power and Energy Society General Meeting, vol. 2018-Augus, pp. 1-5, 2018.

[32] R. Zhang and Y. Xu, "Data-driven dynamic security assessment and control of power systems: An online sequential learning method," Journal of Energy Engineering, vol. 145, no. 5, p. 04019019, 2019.

[33] Q. Li and Y. Xu, "A hybrid data-driven method for online power system dynamic security assessment with incomplete PMU measurements," Proceedings of the IEEE Power \& Energy Society General Meeting (PESGM), no. July, 2019.

[34] Y. Zhou, J. Wu, L. Ji, Z. Yu, K. Lin, and L. Hao, "Transient stability preventive control of power systems using chaotic particle swarm optimization combined with two-stage support vector machine," Electric Power Systems Research, vol. 155, pp. 111-120, 2018. [Online]. Available: http://dx.doi.org/10.1016/j.epsr.2017.10.007

[35] R. Liu, G. Verbic, and Y. Xu, "A new reliability-driven intelligent system for power system dynamic security assessment," 2017 Australasian Universities Power Engineering Conference, AUPEC 2017, vol. 2017Novem, pp. 1-6, 2017

[36] Q. Al-Gubri and M. A. Mohd Ariff, "Real-time power system dynamic security assessment based on advanced feature selection for decision tree classifiers," Turkish Journal of Electrical Engineering and Computer Sciences, vol. 26, no. 4, pp. 2104-2116, 2018.

[37] J. J. Yu, D. J. Hill, A. Y. Lam, J. Gu, and V. O. Li, "Intelligent timeadaptive transient stability assessment system," IEEE Transactions on Power Systems, vol. 33, no. 1, pp. 1049-1058, 2018
[38] B. P. Soni, A. Saxena, V. Gupta, and S. L. Surana, "Assessment of transient stability through coherent machine identification by using least-square support vector machine," Modelling and Simulation in Engineering, vol. 2018, 2018

[39] H. Mohammadi, G. Khademi, M. Dehghani, and D. Simon, "Voltage stability assessment using multi-objective biogeography-based subset selection," International Journal of Electrical Power and Energy Systems, vol. 103, no. June, pp. 525-536, 2018. [Online]. Available: https://doi.org/10.1016/j.ijepes.2018.06.017

[40] N. Tomin, A. Zhukov, D. Sidorov, V. Kurbatsky, D. Panasetsky, and V. Spiryaev, "Random forest based model for preventing large-scale emergencies in power systems," International Journal of Artificial Intelligence, vol. 13, no. 1, pp. 211-228, 2015.

[41] C. Zhang, Y. Li, Z. Yu, and F. Tian, "Feature selection of power system transient stability assessment based on random forest and recursive feature elimination," Asia-Pacific Power and Energy Engineering Conference, APPEEC, vol. 2016-Decem, pp. 1264-1268, 2016.

[42] S. R. Nandanwar and S. Warkad, "Voltage security assessment with application of PMUs using decision tree," in 2016 8th International Conference on Computational Intelligence and Communication Networks (CICN). IEEE, 2016, pp. 365-369.

[43] S. Shen, Q. Liu, X. Tao, and S. Ni, "Application of the XGBOOST on the assessment of transient stability of power system," 2019 International Conference on Electronical, Mechanical and Materials Engineering, vol. 181, no. Ice2me, pp. 6-10, 2019.

[44] H. Mohammadi and M. Dehghani, "PMU based voltage security assessment of power systems exploiting principal component analysis and decision trees," International Journal of Electrical Power and Energy Systems, vol. 64, no. June 2018, pp. 655-663, 2015. [Online]. Available: http://dx.doi.org/10.1016/j.ijepes.2014.07.077

[45] H. Fan, Y. Chen, S. Huang, X. Zhang, H. Guan, and D. Shi, "Post-fault transient stability assessment based on k-nearest neighbor algorithm with mahalanobis distance," in 2018 International Conference on Power System Technology (POWERCON). IEEE, 2018, pp. 44174423 .

[46] Z. Guoli, G. Qingwu, Q. Wenxiao, L. Jianqiang, Z. Bowen, G. He, Z. Tingting, W. Liuchuang, C. Wenhui, L. Xu, W. Bo, and Q. Hui, "Power system transient voltage stability assessment based on kernel principal component analysis and dbn," in International Conference of Artificial Intelligence, Medical Engineering, Education. Springer, 2018, pp. 713-728.

[47] T. Li, J. Liu, K. Gao, J. Tang, D. Cui, H. Zeng, T. Wang, Z. Wang, $\mathrm{Y}$. Zhang, and X. Xu, "Decision tree-based real-time emergency control strategy for power system," in 2018 International Conference on Power System Technology (POWERCON), no. 201804270000933. IEEE, 2018, pp. 1832-1838.

[48] S. Xie, C. Lu, L. Zhu, and J. Zhang, "Online long-term voltage stability assessment based on time series shapelet extraction," International Conference on Innovative Smart Grid Technologies, ISGT Asia 2018 , pp. 1153-1158, 2018.

[49] B. Tan, J. Yang, X. Pan, J. Li, P. Xie, and C. Zeng, "Representational learning approach for power system transient stability assessment based on convolutional neural network," The Journal of Engineering, vol. 2017, no. 13, pp. 1847-1850, 2017.

[50] X. Yin and Y. Liu, "Deep learning based feature reduction for power system transient stability assessment," in TENCON 2018-2018 IEEE Region 10 Conference. IEEE, 2018, pp. 2308-2312.

[51] J. Tang and H. Sui, "Power system transient stability assessment based on stacked autoencoders and support vector machine," in IOP Conference Series: Materials Science and Engineering, vol. 452, no. 4. IOP Publishing, 2018, p. 042117.

[52] Q. Zhu, J. Chen, L. Zhu, D. Shi, X. Bai, X. Duan, and Y. Liu, "A deep end-to-end model for transient stability assessment with PMU data," IEEE Access, vol. 6, pp. 65 474-65 487, 2018.

[53] L. Zhu, C. Lu, Z. Y. Dong, and C. Hong, "Imbalance learning machinebased power system short-term voltage stability assessment," IEEE Transactions on Industrial Informatics, vol. 13, no. 5, pp. 2533-2543, 2017.

[54] J. Hou, C. Xie, T. Wang, Z. Yu, Y. Lü, and H. Dai, "Power system transient stability assessment based on voltage phasor and convolution neural network," Proceedings - 2nd IEEE International Conference on Energy Internet, ICEI 2018, pp. 247-251, 2018.

[55] Y. Zhou, W. Zhao, Q. Guo, H. Sun, and L. Hao, "Transient stability assessment of power systems using cost-sensitive deep learning approach," in 2018 2nd IEEE Conference on Energy Internet and Energy System Integration (EI2). IEEE, 2018, pp. 1-6. 
[56] G. N. Baltas, C. Perales-González, P. Mazidi, and F. Fernandez, "A novel ensemble approach for solving the transient stability classification problem," 7th International Conference on Renewable Energy Research and Applications (ICRERA), vol. 5, pp. 1-5, 2018.

[57] H. T. Nguyen and L. B. Le, "Online ensemble learning for security assessment in PMU based power system," IEEE International Conference on Sustainable Energy Technologies, ICSET, pp. 384-389, 2016.

[58] A. Nath, R. S. Biswas, and A. Pal, "Application of machine learning for online dynamic security assessment in presence of system variability and additive instrumentation errors," Arxiv, 2019.

[59] N. V. Tomin, V. G. Kurbatsky, D. N. Sidorov, and A. V. Zhukov, "Machine learning techniques for power system security assessment," IFAC-PapersOnLine, vol. 49, no. 27, pp. 445-450, 2016. [Online]. Available: http://dx.doi.org/10.1016/j.ifacol.2016.10.773

[60] V. Kurbatsky and N. Tomin, "Identification of pre-emergency states in the electric power system on the basis of machine learning technologies," in 2016 12th World Congress on Intelligent Control and Automation (WCICA). IEEE, 2016, pp. 378-383.

[61] J. Cao and Z. Fan, "Deep learning-based online small signal stability assessment of power systems with renewable generation," Proceedings - 2018 IEEE SmartWorld, Ubiquitous Intelligence and Computing, Advanced and Trusted Computing, Scalable Computing and Communications, Cloud and Big Data Computing, Internet of People and Smart City Innovations, SmartWorld/UIC/ATC/ScalCom/CBDCo, pp. 216-221, 2018.

[62] A. K. Gupta, K. Verma, and K. R. Niazi, "Intelligent wide area monitoring of power system oscillatory dynamics in real time," 2017 4th International Conference on Advanced Computing and Communication Systems, ICACCS 2017, 2017.

[63] S. A. Siddiqui, K. Verma, K. R. Niazi, and M. Fozdar, "Artificial neural network based early detection of real-time transient instability for initiation of emergency control through wide-area synchrophasor measurements," IEEE International Conference on Computer Communication and Control, IC4 2015, no. September, 2015.

[64] B. P. Soni, V. Gupta, R. Kumar, A. Saxena, and S. L. Surana, "Application of ANN for stability assessment of large power system by post-fault rotor angle measurements," 2018 IEEMA Engineer Infinite Conference, eTechNxT 2018, pp. 1-6, 2018.

[65] S. M. Ashraf, A. Gupta, D. K. Choudhary, and S. Chakrabarti, "Voltage stability monitoring of power systems using reduced network and artificial neural network," International Journal of Electrical Power and Energy Systems, vol. 87, pp. 43-51, 2017. [Online]. Available: http://dx.doi.org/10.1016/j.ijepes.2016.11.008

[66] C. Subramani, A. A. Jimoh, S. H. Kiran, and S. S. Dash, "Artificial neural network based voltage stability analysis in power system," in 2016 International Conference on Circuit, Power and Computing Technologies (ICCPCT). IEEE, 2016, pp. 1-4.

[67] Y. Liu, Y. Liu, J. Liu, M. Li, T. Liu, G. Taylor, and K. Zuo, "A mapreduce based high performance neural network in enabling fast stability assessment of power systems," Mathematical Problems in Engineering, vol. 2017, 2017.

[68] M. Li, P. Jiang, W. Wu, R. Ye, Y. Du, H. Zheng, and T. Yan, "Artificial neural network classifier of transient stability based on time-domain simulation," Proceedings of 2018 2nd IEEE Advanced Information Management, Communicates, Electronic and Automation Control Conference, IMCEC 2018, no. Imcec, pp. 685-689, 2018.

[69] P. Beyranvand, V. M. I. Genç, and Z. Çataltepe, "Multilabel learning for the online transient stability assessment of electric power systems," Turkish Journal of Electrical Engineering and Computer Sciences, vol. 26, no. 5, pp. 2661-2675, 2018.

[70] A. B. Mosavi, A. Amiri, and H. Hosseini, "A learning framework for size and type independent transient stability prediction of power system using twin convolutional support vector machine," IEEE Access, vol. 6 , no. c, pp. 69937-69947, 2017.

[71] Y. Zhou, Q. Guo, H. Sun, Z. Yu, J. Wu, and L. Hao, "A novel data-driven approach for transient stability prediction of power systems considering the operational variability," International Journal of Electrical Power and Energy Systems, vol. 107, no. December 2018, pp. 379-394, 2019. [Online]. Available: https://doi.org/10.1016/j.ijepes.2018.11.031

[72] R. Zhang, J. Wu, Y. Xu, B. Li, and M. Shao, "A hierarchical selfadaptive method for post-disturbance transient stability assessment of power systems using an integrated CNN-based ensemble classifier," Energies, vol. 12, no. 17, p. 3217, 2019.

[73] R. Yan, G. Geng, Q. Jiang, and Y. Li, "Fast transient stability batch assessment using cascaded convolutional neural networks," IEEE Transactions on Power Systems, vol. 34, no. 4, pp. 2802-2813, 2019.
[74] J.-M. H. Arteaga, F. Hancharou, F. Thams, and S. Chatzivasileiadis, "Deep learning for power system security assessment," 13th IEEE PowerTech 2019, pp. 1-6, 2019.

[75] Y. Zhang, T. Li, G. Na, G. Li, and Y. Li, "Optimized extreme learning machine for power system transient stability prediction using synchrophasors," Mathematical Problems in Engineering, vol. 2015, 2015.

[76] C. Ren, Y. Xu, and Y. Zhang, "Post-disturbance transient stability assessment of power systems towards optimal accuracy-speed tradeoff," Protection and Control of Modern Power Systems, vol. 3, no. 1, pp. $1-10,2018$.

[77] C. Ren and Y. Xu, "A fully data-driven method based on generative adversarial networks for power system dynamic security assessment with missing data," IEEE Transactions on Power Systems, vol. PP, no. c, pp. 1-1, 2019.

[78] P. Duraipandy and D. Devaraj, "Development of extreme learning machine for online voltage stability assessment incorporating wind energy conversion system," in 2017 IEEE International Conference on Intelligent Techniques in Control, Optimization and Signal Processing (INCOS). IEEE, 2017, pp. 1-7.

[79] Y. Zhang, Y. Xu, Z. Y. Dong, and P. Zhang, "Real-time assessment of fault-induced delayed voltage recovery: A probabilistic self-adaptive data-driven method," IEEE Transactions on Smart Grid, vol. 10, no. 3 , pp. 2485-2494, 2019.

[80] Q. Wang, C. Zhang, L. Ying, Z. Yu, and Y. Tang, "Data inheritancebased updating method and its application in transient frequency prediction for a power system," International Transactions on Electrical Energy Systems, vol. 29, no. 6, pp. 1-16, 2019.

[81] L. Zheng, W. Hu, K. Hou, X. Xu, and G. Shao, "Real-time transient stability assessment based on deep recurrent neural network," 2017 IEEE Innovative Smart Grid Technologies - Asia: Smart Grid for Smart Community, ISGT-Asia 2017, pp. 1-5, 2017.

[82] C. B. Saner, M. Kesici, M. Mahdi, Y. Yaslan, and V. I. Genc, "Wide area measurement-based transient stability prediction using long short-term memory networks," in 2019 7th International Istanbul Smart Grids and Cities Congress and Fair (ICSG). IEEE, 2019, pp. 159-163.

[83] A. Gupta, G. Gurrala, and P. S. Sastry, "Instability prediction in power systems using recurrent neural networks," IJCAI International Joint Conference on Artificial Intelligence, pp. 1795-1801, 2017.

[84] M. Barati, "Faster than real-time prediction of disruptions in power grids using PMU: Gated recurrent unit approach," 2019 IEEE Power \& Energy Society Innovative Smart Grid Technologies Conference, pp. $1-5,2019$.

[85] Z. Pannell, B. Ramachandran, and D. Snider, "Machine learning approach to solving the transient stability assessment problem," in 2018 IEEE Texas Power and Energy Conference (TPEC). IEEE, 2018, pp. $1-6$.

[86] Y. Zhou, H. Sun, Q. Guo, B. Xu, J. Wu, and L. Hao, "Data driven method for transient stability prediction of power systems considering incomplete measurements," in 2017 IEEE Conference on Energy Internet and Energy System Integration (EI2). IEEE, 2017, pp. 1-6.

[87] W. Hu, Z. Lu, S. Wu, W. Zhang, Y. Dong, R. Yu, and B. Liu, "Real-time transient stability assessment in power system based on improved SVM," Journal of Modern Power Systems and Clean Energy, vol. 7, no. 1, pp. 26-37, 2019. [Online]. Available: https://doi.org/10.1007/s40565-018-0453-x

[88] D. E. Echeverría, J. C. Cepeda, and D. Graciela Colomé, "Realtime transient stability assessment of electric power systems using predictive-sime based on machine learning," 2017 IEEE PES Innovative Smart Grid Technologies Conference - Latin America, ISGT Latin America 2017, vol. 2017-Janua, pp. 1-6, 2017.

[89] G. S. Naganathan and C. K. Babulal, "Optimization of support vector machine parameters for voltage stability margin assessment in the deregulated power system," Soft Computing, vol. 23, no. 20, pp. 10495-10 507, 2018. [Online]. Available: https://doi.org/10.1007/ s00500-018-3615-X

[90] Y. Zhou, J. Wu, Z. Yu, L. Ji, and L. Hao, "A hierarchical method for transient stability prediction of power systems using the confidence of a SVM-based ensemble classifier," Energies, vol. 9, no. 10, 2016.

[91] S. S. Maaji, G. Cosma, A. Taherkhani, A. A. Alani, and T. M. McGinnity, "On-line voltage stability monitoring using an ensemble AdaBoost classifier," 2018 4th International Conference on Information Management, ICIM 2018, pp. 253-259, 2018.

[92] A. Hoballah, "Online system stability monitoring using DT and ANN considering stochastic behavior of wind speed," in 2015 4th International Conference on Electric Power and Energy Conversion Systems (EPECS). IEEE, 2015, pp. 1-6. 
[93] P. N. Papadopoulos, J. V. Milanovic, P. Bhui, and N. Senroy, "Fast online identification of power system dynamic behavior," IEEE Power and Energy Society General Meeting, pp. 1-5, 2017.

[94] J. L. Cremer, I. Konstantelos, and G. Strbac, "From optimization-based machine learning to interpretable security rules for operation," IEEE Transactions on Power Systems, vol. 34, no. 5, pp. 3826-3836, 2019.

[95] I. Konstantelos, G. Jamgotchian, S. H. Tindemans, P. Duchesne, S. Cole, C. Merckx, G. Strbac, and P. Panciatici, "Implementation of a massively parallel dynamic security assessment platform for large-scale grids," IEEE Transactions on Smart Grid, vol. 8, no. 3, pp. 1417-1426, 2016.

[96] M. Vasconcelos, L. M. Carvalho, J. Meirinhos, N. Omont, P. GambierMorel, G. Jamgotchian, D. Cirio, E. Ciapessoni, A. Pitto, I. Konstantelos et al., "Online security assessment with load and renewable generation uncertainty: The iTesla project approach," in 2016 International Conference on Probabilistic Methods Applied to Power Systems (PMAPS). IEEE, 2016, pp. 1-8.

[97] Z. Nie, D. Yang, V. Centeno, and K. D. Jones, "A PMU-based voltage security assessment framework using hoeffding-tree-based learning," 2017 19th International Conference on Intelligent System Application to Power Systems, ISAP 2017, no. January 2018, 2017.

[98] H. Suprême, L. A. Dessaint, I. Kamwa, and A. Heniche-Oussédik, "Development of new predictors based on the concept of center of power for transient and dynamic instability detection," IEEE Transactions on Smart Grid, vol. 9, no. 4, pp. 3605-3615, 2016.

[99] Q. A. Al-Gubri, M. A. Ariff, and I. S. Saeh, "Performance analysis of machine learning algorithms for power system dynamic security assessment," IET Conference Publications, vol. 2016, no. CP688, 2016.

[100] J. D. Pinzón and D. G. Colomé, "Real-time multi-state classification of short-term voltage stability based on multivariate time series machine learning," International Journal of Electrical Power and Energy Systems, vol. 108, no. January, pp. 402-414, 2019. [Online]. Available: https://doi.org/10.1016/j.ijepes.2019.01.022

[101] C. Liu, F. Tang, and C. L. Bak, "An accurate online dynamic security assessment scheme based on random forest," Energies, vol. 11, no. 7 , 2018.

[102] T. Dimitrovska, U. Rudež, R. Mihalič, and U. Kerin, "Transient stability contingency screening and ranking based on data mining," IEEE PES Innovative Smart Grid Technologies Conference Europe, 2017.

[103] Q. Yang, G. Wang, A. Sadeghi, G. B. Giannakis, and J. Sun, "Real-time voltage control using deep reinforcement learning," Arxiv, pp. 1-9, 2019. [Online]. Available: http://arxiv.org/abs/1904.09374

[104] J. Sun, Z. Zhu, H. Li, Y. Chai, G. Qi, H. Wang, and Y. H. Hu, "An integrated critic-actor neural network for reinforcement learning with application of DERs control in grid frequency regulation," International Journal of Electrical Power and Energy Systems, vol. 111, no. March, pp. 286-299, 2019. [Online]. Available: https://doi.org/10.1016/j.ijepes.2019.04.011

[105] Y. Tang, H. He, J. Wen, and J. Liu, "Power System stability control for a wind farm based on adaptive dynamic programming," IEEE Transactions on Smart Grid, vol. 6, no. 1, pp. 166-177, 2015.

[106] C. Mu, Y. Tang, and H. He, "Improved sliding mode design for load frequency control of power system integrated an adaptive learning strategy," IEEE Transactions on Industrial Electronics, vol. 64, no. 8, pp. 6742-6751, 2017.

[107] T. Guo and J. V. Milanovic, "Online identification of power system dynamic signature using PMU measurements and data mining," IEEE Transactions on Power Systems, vol. 31, no. 3, pp. 1760-1768, 2016.

[108] D. Yang, Z. Nie, K. Jones, and V. Centeno, "Adaptive decisiontrees-based regional voltage control," in 2017 North American Power Symposium (NAPS). IEEE, 2017, pp. 1-6.

[109] D. Yang, "A power system network partition framework for data-driven regional voltage control," 2017 North American Power Symposium, NAPS 2017, 2017.

[110] N. Tomin, A. Zhukov, V. Kurbatsky, D. Sidorov, and M. Negnevitsky, "Development of automatic intelligent system for on-line voltage security control of power systems," 2017 IEEE Manchester PowerTech, Powertech 2017, 2017.

[111] A. N. Al-Masri, M. Z. Ab Kadir, H. Hizam, and N. Mariun, "Simulation of an adaptive artificial neural network for power system security enhancement including control action," Applied Soft Computing Journal, vol. 29, pp. 1-11, 2015. [Online]. Available: http://dx.doi.org/10.1016/j.asoc.2014.12.006

[112] D. Xu, J. Liu, X. G. Yan, and W. Yan, "A novel adaptive neural network constrained control for a multi-area interconnected power system with hybrid energy storage," IEEE Transactions on Industrial Electronics, vol. 65 , no. 8, pp. 6625-6634, 2017.
[113] A. Karami and K. Mahmoodi Galougahi, "Improvement in power system transient stability by using STATCOM and neural networks," Electrical Engineering, vol. 101, no. 1, pp. 19-33, 2019. [Online] Available: https://doi.org/10.1007/s00202-019-00753-5

[114] Z. Deng and M. Liu, "An integrated generation-compensation optimization strategy for enhanced short-term voltage security of large-scale power systems using multi-objective reinforcement learning method,' 2018 International Conference on Power System Technology, POWERCON 2018 - Proceedings, pp. 4099-4106, 2018.

[115] F. Tian, X. Zhou, Z. Yu, D. Shi, Y. Chen, and Y. Huang, "A preventive transient stability control method based on support vector machine," Electric Power Systems Research, vol. 170, no. January, pp. 286-293, 2019. [Online]. Available: https://doi.org/10.1016/j.epsr.2019.01.030

[116] H. Nguyen-Duc, L. Tran-Hoai, and D. Vo Ngoc, "A novel approach to solve transient stability constrained optimal power flow problems," Turkish Journal of Electrical Engineering and Computer Sciences, vol. 25, no. 6, pp. 4696-4705, 2017.

[117] M. Mokhayeri, M. Shivaie, S. Khazaei, and M. K. Moghaddam, "A phasor-based framework for real-time identification of power systems islanding through decision-tree algorithm," 2018 Smart Grid Conference (SGC), pp. 1-8, 2018.

[118] J. L. Cremer, I. Konstantelos, G. Strbac, and S. H. Tindemans, "Samplederived disjunctive rules for secure power system operation," in 2018 IEEE International Conference on Probabilistic Methods Applied to Power Systems (PMAPS). IEEE, 2018, pp. 1-6.

[119] J. L. Cremer, I. Konstantelos, S. H. Tindemans, and G. Strbac, "Datadriven power system operation: Exploring the balance between cost and risk," IEEE Transactions on Power Systems, vol. 34, no. 1, pp. 791-801, 2019.

[120] F. Thams, L. Halilbasic, P. Pinson, S. Chatzivasileiadis, and R. Eriksson, "Data-driven security-constrained OPF," Proceedings of the 10th Bulk Power Systems Dynamics and Control Symposium, 2017.

[121] L. Halilbašić, F. Thams, A. Venzke, S. Chatzivasileiadis, and P. Pinson, "Data-driven security-constrained AC-OPF for operations and markets," in 2018 Power Systems Computation Conference (PSCC). IEEE, 2018, pp. 1-7.

[122] H. Sun, F. Zhao, H. Wang, K. Wang, W. Jiang, Q. Guo, B. Zhang, and L. Wehenkel, "Automatic learning of fine operating rules for online power system security control," IEEE Transactions on Neural Networks and Learning Systems, vol. 27, no. 8, pp. 1708-1719, 2015.

[123] V. Malbasa, C. Zheng, P. C. Chen, T. Popovic, and M. Kezunovic, "Voltage stability prediction using active machine learning," IEEE Transactions on Smart Grid, vol. 8, no. 6, pp. 3117-3124, 2017.

[124] B. Donnot, I. Guyon, M. Schoenauer, P. Panciatici, and A. Marot, "Introducing machine learning for power system operation support," Arxiv, 2017. [Online]. Available: http://arxiv.org/abs/1709.09527

[125] B. Donnot, I. Guyon, M. Schoenauer, A. Marot, and P. Panciatici, "Fast power system security analysis with guided dropout," 26th European Symposium on Artificial Neural Networks (ESAAN), Bruges, April 2018. [Online]. Available: http://arxiv.org/abs/1801.09870

[126] — "Anticipating contingengies in power grids using fast neural net screening," Proceedings of the International Joint Conference on Neural Networks, vol. 2018-July, 2018.

[127] B. Donnot, I. Guyon, A. Marot, M. Schoenauer, and P. Panciatici, "Optimization of computational budget for power system risk assessment," Proceedings - 2018 IEEE PES Innovative Smart Grid Technologies Conference Europe, ISGT-Europe 2018, no. 1, pp. 1-6, 2018.

[128] B. Donnot, B. Donon, I. Guyon, Z. Liu, A. Marot, P. Panciatici, and M. Schoenauer, "LEAP nets for power grid perturbations," European Symposium on Artificial Neural Networks, Computational Intelligence and Machine Learning, no. April, pp. 24-26, 2019. [Online]. Available: http://arxiv.org/abs/1908.08314

[129] D. Abdellah and L. Djamel, "Power flow analysis using adaptive neurofuzzy inference systems," in 2015 3rd International Renewable and Sustainable Energy Conference (IRSEC). IEEE, 2015, pp. 1-5.

[130] N. G. Singhal, N. Li, and K. W. Hedman, "A data-driven reserve response set policy for power systems with stochastic resources," IEEE Transactions on Sustainable Energy, vol. 10, no. 2, pp. 693-705, 2018.

[131] M. K. Paramathma, D. Devaraj, and B. S. Reddy, "Artificial neural network based static security assessment module using PMU measurements for smart grid application," 1st International Conference on Emerging Trends in Engineering, Technology and Science, ICETETS 2016 - Proceedings, pp. 1-5, 2016.

[132] I. Bhatt, A. Dhandhia, and V. Pandya, "Static security assessment of power system using radial basis function neural network module," in 2017 IEEE International WIE Conference on Electrical and Computer Engineering (WIECON-ECE). IEEE, 2017, pp. 274-278. 
[133] M. Lekshmi and M. S. Nagaraj, "Online static security assessment module using radial basis neural network trained with particle swarm optimization," Lecture Notes in Electrical Engineering, vol. 446, pp. 215-224, 2018.

[134] Y. Li, Y. Li, and Y. Sun, "Online static security assessment of power systems based on Lasso algorithm," Applied Sciences (Switzerland), vol. 8, no. 9, 2018.

[135] M. Gholami, G. B. Gharehpetian, and M. Mohammadi, "Intelligent hierarchical structure of classifiers to assess static security of power system," Journal of Intelligent and Fuzzy Systems, vol. 28, no. 6, pp. 2875-2880, 2015

[136] I. S. Saeh, M. W. Mustafa, Y. S. Mohammed, and M. Almaktar, "Static security classification and evaluation classifier design in electric power grid with presence of PV power plants using C-4 . 5," Renewable and Sustainable Energy Reviews, vol. 56, pp. 283-290, 2016. [Online]. Available: http://dx.doi.org/10.1016/j.rser.2015.11.054

[137] H. Y. Su and T. Y. Liu, "Enhanced-online-random-forest model for static voltage stability assessment using wide area measurements," IEEE Transactions on Power Systems, vol. 33, no. 6, pp. 6696-6704, 2018.

[138] A. Zhukov, N. Tomin, V. Kurbatsky, D. Sidorov, D. Panasetsky, and A. Foley, "Ensemble methods of classification for power systems security assessment," Applied Computing and Informatics, vol. 15, no. 1, pp. 45-53, 2017. [Online]. Available: https://doi.org/10.1016/j. aci.2017.09.007http://dx.doi.org/10.1016/j.aci.2017.09.007

[139] M. Sun, I. Konstantelos, and G. Strbac, "A deep learning-based feature extraction framework for system security assessment," IEEE Transactions on Smart Grid, vol. PP, no. c, p. 1, 2018.

[140] W. D. Oliveira, J. P. Vieira, U. H. Bezerra, D. A. Martins, and B. d. G. Rodrigues, "Power system security assessment for multiple contingencies using multiway decision tree," Electric Power Systems Research, vol. 148, pp. 264-272, 2017. [Online]. Available: http://dx.doi.org/10.1016/j.epsr.2017.03.029

[141] A. Dhandhia, V. Pandya, and P. Bhatt, "Multi-class support vector machines for static security assessment of power system," Ain Shams Engineering Journal, no. xxxx, 2019. [Online]. Available: https://doi.org/10.1016/j.asej.2019.07.011

[142] V. Thirugnanasambandam and T. Jain, "AdaBoost classifiers for phasor measurements-based security assessment of power systems," IET Generation, Transmission and Distribution, vol. 12, no. 8, pp. 1747-1755, 2018.

[143] K. Chakraborty and G. Saha, "Off-line voltage security assessment of power transmission systems using uvsi through artificial neural network," in 2016 International Conference on Intelligent Control Power and Instrumentation (ICICPI). IEEE, 2016, pp. 158-162.

[144] P. Singh, L. S. Titare, L. D. Arya, and S. C. Choube, "On-line probabilistic voltage security assessment using Radial Basis Function neural network," Proceedings of 2016 International Conference on ICT in Business, Industry, and Government, ICTBIG 2016, 2016.

[145] Z. Yun, Q. Zhou, Y. Feng, D. Sun, J. Sun, and D. Yang, "On-line static voltage security risk assessment based on Markov chain model and SVM for wind integrated power system," in 2017 13th International Conference on Natural Computation, Fuzzy Systems and Knowledge Discovery (ICNC-FSKD). IEEE, 2017, pp. 2469-2473.

[146] W. Li, P. Zhang, S. Su, X. Meng, C. Ding, and Y. Wang, "Comparison of decision tree attribute selection methods for static voltage stability margin assessment," 2018 IEEE 2nd International Electrical and Energy Conference, pp. 201-206, 2018.

[147] J. D. A. Colorado, S. P. Londoño, and J. J. M. Flórez, "Identifying voltage stability critical areas using k-means clustering technique," 2016 IEEE PES Transmission and Distribution Conference and ExpositionLatin America, PES T and D-LA 2016, no. 1, 2016.

[148] T. E. Huang, Q. Guo, H. Sun, C. W. Tan, and T. Hu, "A deep spatial-temporal data-driven approach considering microclimates for power system security assessment," Applied Energy, vol. 237, no. January, pp. 36-48, 2019. [Online]. Available: https: //doi.org/10.1016/j.apenergy.2019.01.013

[149] S. M. Pérez-Londoño, G. Olivar-tost, and J. J. Mora-florez, "Online determination of voltage stability weak areas for situational awareness improvement," Electric Power Systems Research, vol. 145, pp. 112 121, 2017

[150] S. Dadi, S. Mathew, and V. V. Khatavkar, "Estimation of transmission reliability margin using statistical methods and intelligent techniques," International Conference on Automatic Control and Dynamic Optimization Techniques, ICACDOT 2016, pp. 896-900, 2016.
[151] G. Qiu, J. Liu, Y. Liu, T. Liu, and G. Mu, "Ensemble learning for power systems TTC prediction with wind farms," IEEE Access, vol. 7, pp. 16572-16583, 2019.

[152] Y. Fan, X. Li, and P. Zhang, "Real-time static voltage stability assessment in large-scale power systems based on maximum-relevance minimum-redundancy ensemble approach," IEEE Access, vol. 5, pp. $27281-27291,2017$.

[153] X. Pan, T. Zhao, and M. Chen, "DeepOPF: deep neural network for DC optimal power flow," Arxiv, 2019. [Online]. Available: https://arxiv.org/pdf/1905.04479.pdf

[154] F. Fioretto, T. W. K. Mak, and P. Van Hentenryck, "Predicting AC optimal power flows: combining deep learning and lagrangian dual methods," Arxiv, no. 2, 2019. [Online]. Available: http: //arxiv.org/abs/1909.10461

[155] A. Zamzam and K. Baker, "Learning optimal solutions for extremely fast AC optimal power flow," Arxiv, pp. 1-7, 2019. [Online]. Available: http://arxiv.org/abs/1910.01213

[156] Y. Sun, X. Fan, Q. Huang, X. Li, R. Huang, T. Yin, and G. Lin, "Local feature sufficiency exploration for predicting security-constrained generation dispatch in multi-area power systems," in 2018 17th IEEE International Conference on Machine Learning and Applications (ICMLA). IEEE, 2018, pp. 1283-1289.

[157] K. Baker, "Learning warm-start points for AC optimal power low," Arxiv, 2019. [Online]. Available: http://arxiv.org/abs/1905.08860

[158] R. Canyasse, G. Dalal, and S. Mannor, "Supervised learning for optimal power flow as a real-time proxy," 2017 IEEE Power and Energy Society Innovative Smart Grid Technologies Conference, ISGT 2017, 2017.

[159] L. Duchesne, E. Karangelos, and L. Wehenkel, "Machine learning of real-time power systems reliability management response," 2017 IEEE Manchester PowerTech, Powertech 2017, 2017.

[160] — - "Using machine learning to enable probabilistic reliability assessment in operation planning," in 2018 Power Systems Computation Conference (PSCC). IEEE, 2018, pp. 1-8.

[161] D. Urgun and C. Singh, "Multi label RBF classification method for composite system reliability evaluation," 2018 International Conference on Probabilistic Methods Applied to Power Systems, PMAPS 2018 - Proceedings, 2018.

[162] _ _ "A hybrid monte carlo simulation and multi label classification method for composite system reliability evaluation," IEEE Transactions on Power Systems, vol. 34, no. 2, pp. 908-917, 2019.

[163] A. J. Ardakani and F. Bouffard, "Prediction of umbrella constraints," in 2018 Power Systems Computation Conference (PSCC). IEEE, 2018 , pp. 1-7.

[164] D. Deka and S. Misra, "Learning for DC-OPF: Classifying active sets using neural nets," Arxiv, pp. 1-6, 2019.

[165] Y. Ng, S. Misra, L. A. Roald, and S. Backhaus, "Statistical learning for DC optimal power flow," 20th Power Systems Computation Conference, PSCC 2018, pp. 1-7, 2018

[166] S. Misra, L. Roald, and Y. Ng, "Learning for constrained optimization: Identifying optimal active constraint sets," Arxiv, pp. 1-16, 2018. [Online]. Available: http://arxiv.org/abs/1802.09639

[167] I. Mezghani, S. Misra, and D. Deka, "Stochastic AC optimal power flow: a data-driven approach," Arxiv, pp. 1-8, 2019. [Online]. Available: http://arxiv.org/abs/1910.09144

[168] G. Dalal, E. Gilboa, S. Mannor, and L. Wehenkel, "Unit commitment using nearest neighbor as a short-term proxy," 20th Power Systems Computation Conference, PSCC 2018, 2018.

[169] _ - "Chance-constrained outage scheduling using a machine learning proxy," IEEE Transactions on Power Systems, vol. 34, no. 4, pp. 2528$2540,2019$.

[170] G. Dalal, G. Gilboa, and S. Mannor, "Hierarchical decision making in electricity grid management," 33rd International Conference on Machine Learning, ICML 2016, vol. 5, pp. 3249-3258, 2016.

[171] P. Jain, V. Batra, and S. J. Darak, "Improved hierarchical decision making policy for reliable and green electricity grid," in 2018 10th International Conference on Communication Systems \& Networks (COMSNETS). IEEE, 2018, pp. 450-453.

[172] G. Dalal and S. Mannor, "Reinforcement learning for the unit commitment problem," 2015 IEEE Eindhoven PowerTech, PowerTech 2015, 2015.

[173] M. Zhou, B. Wang, T. Li, and J. Watada, "A data-driven approach for multi-objective unit commitment under hybrid uncertainties," Energy, vol. 164, pp. 722-733, 2018. [Online]. Available: https: //doi.org/10.1016/j.energy.2018.09.008

[174] B. Wang, M. Zhou, B. Xin, X. Zhao, and J. Watada, "Analysis of operation cost and wind curtailment using multi-objective unit commitment with battery energy storage," Energy, vol. 178, pp. 
101-114, 2019. [Online]. Available: https://doi.org/10.1016/j.energy. 2019.04.108

[175] S. Pineda, J. M. Morales, and A. Jiménez-Cordero, "Data-driven network reduction for transmission-constrained unit commitment," Arxiv, no. 2, pp. 1-8, 2019. [Online]. Available: http://arxiv.org/abs/ 1907.04694

[176] O. P. Mahela, A. G. Shaik, and N. Gupta, "A critical review of detection and classification of power quality events," Renewable and Sustainable Energy Reviews, vol. 41, pp. 495-505, 2015.

[177] K. Chen, C. Huang, and J. He, "Fault detection, classification and location for transmission lines and distribution systems: a review on the methods," High voltage, vol. 1, no. 1, pp. 25-33, 2016.

[178] L. Zhang, G. Wang, and G. B. Giannakis, "Real-time power system state estimation and forecasting via deep unrolled neural networks," IEEE Transactions on Signal Processing, vol. 67, no. 15, pp. 40694077, 2019.

[179] K. Dehghanpour, Z. Wang, J. Wang, Y. Yuan, and F. Bu, “A survey on state estimation techniques and challenges in smart distribution systems," IEEE Transactions on Smart Grid, vol. 10, no. 2, pp. 2312 2322, 2018.

[180] T. Hong and S. Fan, "Probabilistic electric load forecasting: A tutorial review," International Journal of Forecasting, vol. 32, no. 3, pp. 914 938, 2016

[181] C. Voyant, G. Notton, S. Kalogirou, M.-L. Nivet, C. Paoli, F. Motte, and A. Fouilloy, "Machine learning methods for solar radiation forecasting: A review," Renewable Energy, vol. 105, pp. 569-582, 2017.

[182] H. Wang, Z. Lei, X. Zhang, B. Zhou, and J. Peng, "A review of deep learning for renewable energy forecasting," Energy Conversion and Management, vol. 198, p. 111799, 2019.

[183] Y. Chen, Y. Tan, and D. Deka, "Is machine learning in power systems vulnerable?" in 2018 IEEE International Conference on Communications, Control, and Computing Technologies for Smart Grids (SmartGridComm). IEEE, 2018, pp. 1-6.

[184] D. Amodei, C. Olah, J. Steinhardt, P. Christiano, J. Schulman, and D. Mané, "Concrete problems in AI safety," arXiv preprint arXiv:1606.06565, 2016.

Laurine Duchesne graduated in Electrical Engineering in 2016 at the University of Liège. She is currently pursuing the $\mathrm{Ph} . \mathrm{D}$ degree at the same university. Her research interests include power systems reliability, short-term operation and machine learning applications.

Efthymios Karangelos (M'13) received the Diploma degree in mechanical engineering from the National Technical University of Athens, Athens, Greece, in 2005, and the M.Sc. degree in power systems engineering and economics and the Ph.D. degree in electrical engineering from the University of Manchester, Manchester, U.K., in 2007 and 2012, respectively. In 2012, he joined the Department of Electrical Engineering and Computer Science, University of Liège, Liège, Belgium, as a Post-Doctoral Researcher. His current research interests include power system modeling, reliability and risk management and stochastic optimization.

Louis Wehenkel graduated in Electrical Engineering (Electronics) in 1986 and received the Ph.D. degree in 1990, both from the University of Liège, where he is full Professor of Electrical Engineering and Computer Science. His research interests lie in the fields of stochastic methods for systems control, optimization, machine learning and data mining, with applications in complex systems, in particular large scale power systems planning, operation and control, industrial process control, bioinformatics and computer vision. 